\title{
Wide-area photometric and astrometric (Gaia DR2) study of the young cluster NGC 6530 *
}

\author{
F. Damiani, L. Prisinzano, G. Micela, and S. Sciortino
}

\begin{abstract}
INAF - Osservatorio Astronomico di Palermo G.S.Vaiana, Piazza del Parlamento 1, 90134 Palermo, Italy e-mail: damiani@astropa.inaf.it
\end{abstract}

Received 16 July 2018 / Accepted 18 December 2018

\begin{abstract}
Context. NGC 6530 is a young cluster, with a complex morphology and star-formation history. We present a statistical study of its global properties, using a new, large list of candidate members down to masses of 0.2-0.4 $M_{\odot}$ and Gaia DR2 astrometry.

Aims. We consider a larger sky region compared to previous studies, to investigate the entire cluster until its periphery, including any diffuse population all around the main cluster. We study the distribution of extinction and age across the different regions, and obtain constraints on the star-formation history. We also study the dynamics of cluster members.

Methods. Cluster membership was determined on the basis of literature X-ray data, H $\alpha$ emission, near-IR and UV excesses from the VPHAS+ and UKIDSS photometric surveys and published near-IR catalogs, and Gaia DR2 astrometry; moreover, we used a method for photometric selection of M-type pre-main-sequence cluster members, which we recently developed and used for other star-formation regions. The list of candidates includes nearly 3700 stars, of which we estimate approximately 2700 to be genuine NGC 6530 members.

Results. Using Gaia parallaxes, the cluster distance is found to be $1325 \mathrm{pc}$, with errors of $0.5 \%$ (statistical) and $8.5 \%$ (systematic), in agreement with previous determinations. The cluster morphology and boundaries are established with great confidence, from the agreement between the subsamples of members selected using different criteria. There is no diffuse population of members around the cluster, but there are minor condensations of true members in addition to the two main groups in the cluster core and in the Hourglass nebula. Two such subgroups are spatially associated with the stars $7 \mathrm{Sgr}$ (F2II-III) and HD 164536 (O7.5V). There is a definite pattern of sequential star formation across the cluster, within an age range from less than $0.5 \mathrm{Myr}$ to $\sim 5 \mathrm{Myr}$. Extinction is spatially nonuniform, with part of the population still embedded or obscured by thick dust. The precise Gaia proper motion data indicate that the NGC 6530 parent cloud collided with the Galactic plane around $4 \mathrm{Myr}$ ago, and we suggest that event as the trigger of the bulk of star formation in NGC 6530. The internal cluster dynamics is also partially resolved by the Gaia data, indicating expansion of the main cluster population with respect to its center.
\end{abstract}

Key words. open clusters and associations: individual: NGC 6530 - stars: pre-main sequence - parallaxes - proper motions X-rays: stars

\section{Introduction}

The young cluster NGC 6530 is only a few Myr old, associated to the $\mathrm{H}_{\mathrm{II}}$ region $\mathrm{M} 8$ (Lagoon nebula), which was studied using a variety of methods in the past decades (see Tothill et al. 2008 for a review). Its low-mass stars are still found in their premain sequence (PMS) evolutionary phase, and therefore they are bright X-ray sources due to their active coronae (e.g., Feigelson \& Montmerle 1999), show often $\mathrm{H} \alpha$ emission lines and UV excess emission from accretion of matter onto the star surface, and near-IR (NIR) emission from circumstellar dusty disks. All these characteristics have helped to detect a large population of cumulatively 2500 candidate cluster members down to subsolar masses (e.g., 1100 members were found using X-rays and NIR excesses by Damiani et al. 2004, 2006a, and Prisinzano et al. 2005, 2007; 232 members found using Spitzer data by Kumar \& Anandarao 2010; 2427 candidate members found using deeper Chandra X-ray data by Kuhn et al. 2013, 2014, 2015; 235 members found using photometric $u$ and $\mathrm{H} \alpha$

* Full Table 1 is only available at the CDS via anonymous ftp to cdsarc.u-strasbg. fr $(130.79 .128 .5)$ or via http://cdsarc. u-strasbg.fr/viz-bin/qcat?J/A+A/623/A25 data by Kalari et al. 2015), later confirmed by spectroscopic studies (Prisinzano et al. 2012, and in prep.). The member stars exhibit a well-defined spatial structure, with strong indications that some regions (M8-East, Hourglass nebula) are younger than the bulk of the population. M8-East and the Hourglass nebula are associated with the Lagoon nebula and are therefore assumed to lie at the same distance. A similar complexity was also found from the analysis of the large-scale kinematics of the ionized and neutral gas (Damiani et al. 2017a) using several optical emission and absorption lines. Using X-ray membership and deep optical photometry, Prisinzano et al. (2005) redetermined the cluster distance to be $1250 \mathrm{pc}$, sensibly lower than previous values found in the literature $(1800 \mathrm{pc}$, Sung et al. 2000, van den Ancker et al. 1997). The average optical extinction is $A_{V}=1.085$ mag from Sung et al. (2000).

The cluster member list obtained from all these studies cannot however be considered complete, since each of the methods used suffers from some bias, either inherent to its nature or dictated by the availability and quality of observational data. For example, X-ray imaging data obtained with the Chandra X-ray Observatory (Damiani et al. 2004; Kuhn et al. 2013) consist of only two pointings with the ACIS-I CCD imaging spectrometer, with a square field-of-view (FOV) of $17^{\prime}$ on a side. The 
two pointings are of very different exposure time (one of $\sim 60 \mathrm{ks}$ centered on cluster core, the other of $\sim 180 \mathrm{ks}$ centered on the Hourglass nebula). Inside the ACIS FOV, the limiting sensitivity is not uniform, degrading rapidly to $1 / 4$ of its on-axis value near the FOV borders, because of the degradation of the PSF width with off-axis angle. On the other hand, the relatively hard $\mathrm{X}$-ray emission from PMS low-mass stars is relatively insensitive to absorption, which becomes a distinct advantage in the most obscured parts of the cluster. However, since X-ray luminosity in this age range scales in proportion to stellar bolometric luminosity, the lowest stellar masses are only detected in the deepest X-ray observations.

Member selection methods based on $\mathrm{H} \alpha$ emission, NIR and UV excess of PMS stars are by definition only selecting stars with strong lines from disk-accretion phenomena - so-called Classical T Tauri Stars (CTTS) - since the accreted matter becomes hot enough to emit strongly in the UV continuum and in the Balmer lines, or stars with massive circumstellar dusty disks which emit in the NIR more than the stellar photosphere (Class I/II stars). However, these are as a rule only one component (often a secondary one) of the total population of even very young clusters, the rest of the members belonging to the Weak-line T Tauri Star (WTTS, or Class III) population.

We have recently developed a method (Damiani 2018; Paper I), which relies on the specific properties of PMS M stars, to select candidate low-mass members of PMS clusters using deep multiband photometry, such as is becoming available from the several wide area surveys in the optical and NIR (e.g., in the optical: VPHAS+, Drew et al. 2014; PanSTARRS, Chambers et al. 2016; in the NIR: UKIDSS, Lawrence et al. 2007; VVV, Minniti et al. 2011). The method was tested on the Sco OB1 star-forming complex, where approximately $4000 \mathrm{M}$ type candidate members were found in Paper I, and also in the Vela Molecular Ridge region (Prisinzano et al. 2018) and NGC 2264 cluster (Venuti et al. 2019). This method is unbiased with respect to disk- and accretion-related excess emission (or is maybe slightly biased against them, thus becoming complementary to those methods). Since this method requires photometry in the optical $r$ and $i$ bands (besides one of $g, J$, or $H$ bands), it becomes less powerful for extincted regions, so that again there is some complementarity with X-ray imaging. The limitation inherent to the method, of selecting exclusively M-type members, still makes it very powerful, since these stars constitute the majority of the population of any cluster. We therefore examine here if any sizable population of M stars is present in NGC 6530, and the constraints it adds to the cluster structure and formation history.

Finally, the recently released astrometric data (DR2) from the Gaia mission (Gaia Collaboration 2016, 2018) provide us with an additional method for finding candidate members of NGC 6530, on the basis of their parallax and proper motion. Since the precision of both quantities decreases toward fainter magnitudes, astrometric selection becomes increasingly ineffective toward the lowest stellar masses. Both the astrometric and photometric data used here are available over a wide sky area, thus enabling us to study the cluster together with its surroundings.

The purpose of this work is to assemble a most comprehensive list of candidate members of NGC 6530 using the various methods described above, to use this dataset to study global properties of NGC 6530, and to study to what extent those methods are complementary to one another.

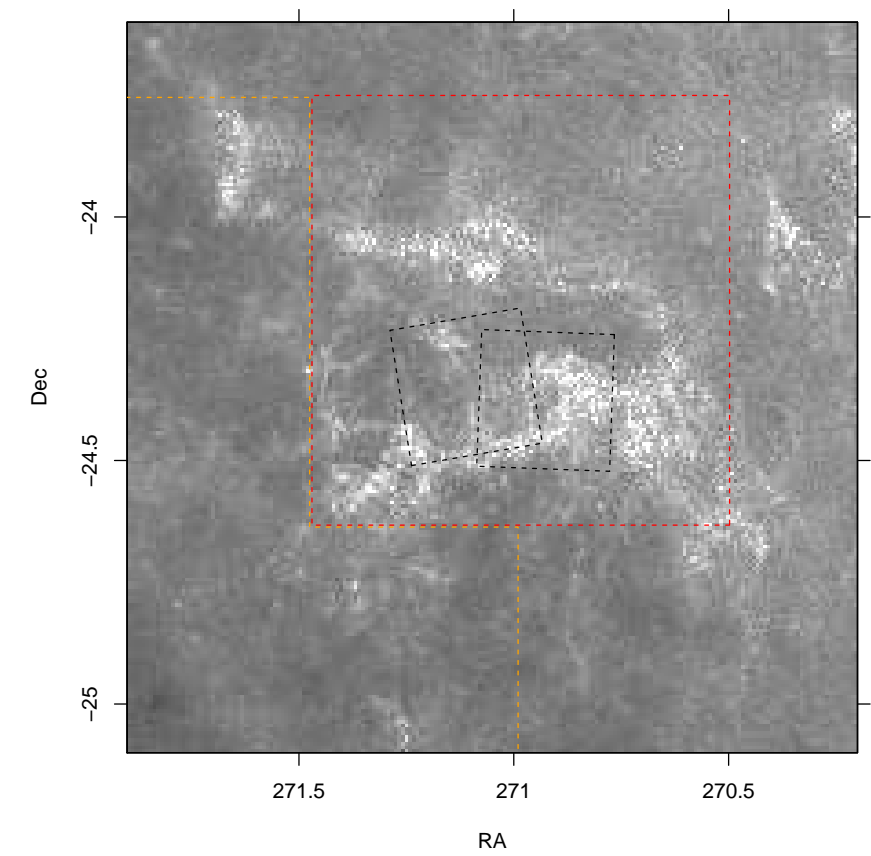

Fig. 1. Spatial density of all Gaia sources in the region studied. Lightgray corresponds to the lowest source density. The two dashed black squares are the Chandra ACIS-I FOVs. The dashed red rectangle indicates the sky region with NIR data from King et al. (2013), while the southeastern region delimited by the dashed orange line has only VVV NIR data.

This paper is structured as follows: in Sect. 2 we describe the observational data used. In Sect. 3 the various methods to select candidate members are presented. Results are studied in Sect. 4, and Sect. 5 provides a conclusive discussion.

\section{Observational data}

This study covers a square sky region of about $1.5^{\circ}$ on a side, $(270.2<$ RA $<271.9 ;-25.1<$ Dec $<-23.6)$ including the NGC 6530 cluster and the associated Lagoon nebula in their entirety, as well as a wide surrounding region. This choice enabled us to search for a possible diffuse population of the cluster, in addition to that found in its densest regions. It was also useful in establishing, through the study of the extinction spatial pattern, the shape and boundaries of the cloud which originated the cluster, and its environment. This star-formation region is a known "blister", excavated by winds and radiation from its massive stars (e.g., Damiani et al. 2017a). This type of geometry is also suggested by the extinction pattern, which is seen as a lower star density in correspondence to thicker dust, for instance in the Gaia (DR2) source catalog whose spatial distribution is shown in Fig. 1. In the same figure we show the Chandra FOVs, which only covered the central regions: cluster core (left-hand FOV) and Hourglass (right-hand FOV). Near the center of the Hourglass region, the Gaia source density is lowest since the dust has the largest column density and obscures background stars; the Hourglass nebula is also a bright diffuse nebula, which causes the optical limiting magnitude to shift toward brighter values. In general, however, the star-density pattern does not follow the shape of the bright nebulosity, so that we conclude that the latter has a minor effect on apparent star density compared to dust extinction. 

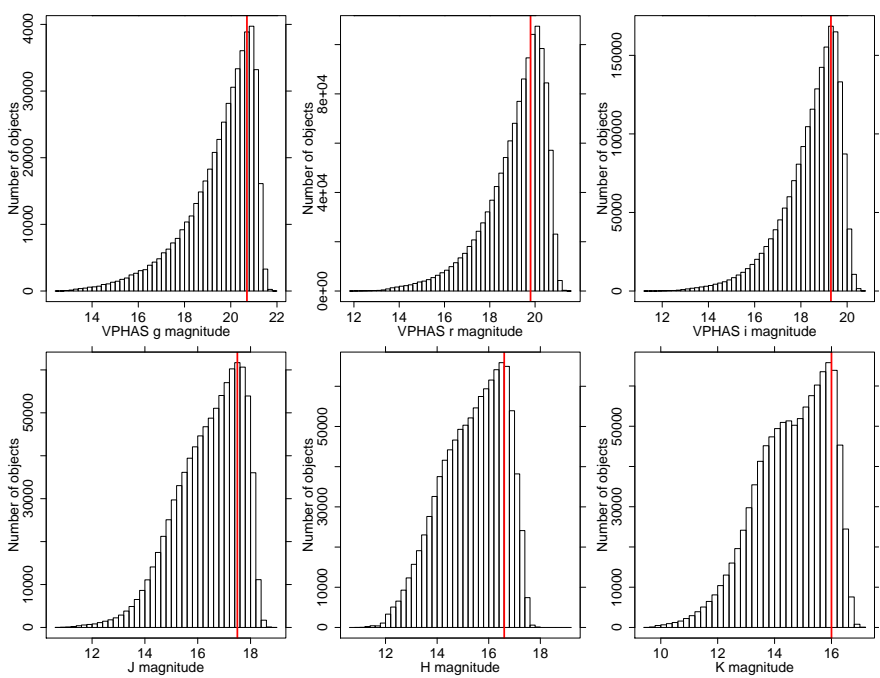

Fig. 2. Magnitude histograms for VPHAS+ bands $g, r, i$ (top panels) and NIR bands $J, H, K$ (bottom panels). The vertical red lines indicate our adopted completeness limits.

\subsection{Optical and NIR photometry}

We used photometric data from the VPHAS+ survey of the Galactic plane, in the $u, g, r, i$ and (narrow) $\mathrm{H} \alpha$ bands. The VPHAS+ (DR2) spatial coverage of the studied region is complete, and the number of VPHAS + objects found is 2109762 . As in previous works (e.g., Kalari et al. 2015; Damiani et al. 2016, Paper I), we used the $r-\mathrm{H} \alpha$ index to select accreting PMS stars with strong $\mathrm{H} \alpha$ lines, while the $u-g$ index was used to find PMS stars with UV-excesses arising from the accretion spot. Magnitude histograms and completeness limits for the $g, r$, and $i$ bands ( $g=20.7, r=19.8, i=19.3$ ) are shown in Fig. 2 . The bright limit for VPHAS+ data is $i \sim 12$, corresponding to cluster stars with masses $\sim 3-4 M_{\odot}$. The few members brighter than this limit, and missed using this photometric set, were however recovered using X-ray or Gaia data (see below). VPHAS + sources were only considered if they have a clean flag set for at least one band; magnitude values without a clean flag set for the given band were not used.

The assembly of our NIR catalog (in the $J H K$ bands) was not as simple as for VPHAS+: the region is not completely covered by the UKIDSS Galactic plane Survey, while 2MASS is too shallow for the purpose of detecting M stars at the NGC 6530 distance. The central parts of the studied region (see Fig. 1), however, were observed by King et al. (2013) using WFCAM at UKIRT, that is the same instrumentation used for UKIDSS surveys; therefore we used the catalog from King et al. (2013). We did not use magnitude values with listed errors larger than $0.3 \mathrm{mag}$, nor with flags equal to "S", "F", "N", "E", or "M". The resulting NIR catalog contains 614787 objects, uniformly covering the region between $270.4977<$ RA $<271.4697$ and $-24.63271<$ Dec $<-23.7505$. In the region covered by both the King et al. (2013) and the UKIDSS catalogs we matched both catalogs to compare their properties. Using the 157811 sources common to both catalogs, we found no significant difference in the respective magnitude values. Magnitude errors in King et al. (2013) were however significantly smaller than in UKIDSS. Therefore, for the spatial region covered by both, we used the King et al. (2013) catalog. Outside that region we used instead the UKIDSS catalog (303481 entries), keeping only (star-like) sources with flag mergedClass $=-1$ or -2 , and magnitudes with flags jppErrBits $<255$, hppErrBits $<255$, and $k \_1 p p E r r B i t s<255$, for the $J, H$ and $K$ bands respectively.

Still, the southeastern part of the whole studied region (Fig. 1) remain uncovered by the combined King-UKIDSS NIR catalog. This region is (almost) completely covered by the VVV survey in the same bands, so we adopted this latter catalog for the remaining spatial region. We found a systematic offset between the UKIDSS and VVV $H$ and $K$ magnitude values (see Appendix A), so before merging the catalogs we subtracted $0.05 \mathrm{mag}$ from the VVV $H$ magnitudes, and $0.02 \mathrm{mag}$ from the VVV $K$ magnitudes. On average, magnitude errors are much larger in the VVV catalog than in UKIDSS, for the common objects in the studied field. We filtered rather heavily the VVV catalog, as described in Paper I; we kept 192541 sources in the region of interest. Magnitude histograms and completeness limits $(J=17.5, H=16.6, K=16.0)$ for the King-UKIDSS NIR catalog are also shown in Fig. 2. This King-UKIDSS-VVV catalog contains 1110809 entries.

For bright NIR sources all the above catalogs are saturated. Therefore, we used the 2MASS catalog (275607 sources in the whole region), filtered as in Paper I, and matched it within 0.2 arcsec with the King-UKIDSS-VVV catalog. The number of unique NIR sources in the obtained King-UKIDSSVVV-2MASS catalog (NIR catalog for brevity) is 1265426. Before merging, we added small zero-point offsets to the KingUKIDSS-VVV magnitudes, for better matching with 2MASS magnitudes, of $+0.0675 \mathrm{mag}$ in $J,-0.043 \mathrm{mag}$ in $H$, and -0.0033 mag in $K$.

By matching the NIR and the optical catalogs within 0.2 arcsec we obtained our final optical-NIR catalog, with 2909548 unique entries. Of them, 470178 sources have both optical and NIR photometry.

\subsection{Astrometric data}

Gaia DR2 data include position, proper motion, parallax, and magnitudes in three bands $(G, B P, R P)$ for 588823 sources in the studied region, distributed in space as shown in Fig. 1. They were matched as above with the optical-NIR catalog, yielding 2950266 unique optical-NIR-Gaia sources. The number of sources common to Gaia and the optical-NIR catalog is 548105. We applied no filtering to the Gaia data at this stage.

\subsection{X-ray data}

We used also X-ray data from the Chandra X-ray Observatory. As mentioned above, Chandra observed two FOVs in the Lagoon nebula (Fig. 1). Results from the "cluster core" field (ObsID 977, 60 ks exposure time) were published by Damiani et al. (2004), while the combined analysis of this same dataset and the much deeper Hourglass field (ObsIDs 3754, 4397, and 4444, sharing the same FOV and totalling $180 \mathrm{ks}$ exposure) was performed as part of the MYStIX project, among several other massive star-formation regions (Feigelson et al. 2013; Kuhn et al. 2013). In general, choices regarding source detection thresholds are more conservative in Damiani et al. (2004) than in the MYStIX papers; in the latter, the existence of very faint $\mathrm{X}$-ray detections almost requires an independent confirmation from identification with another catalog (typically in the NIR). The fact that the Hourglass Chandra field is much deeper than the cluster-core field also means that X-ray sources in the former are overrepresented, and may give a biased picture of the actual stellar density distribution across subregions in the Lagoon nebula because of the spatially non-uniform depth. 

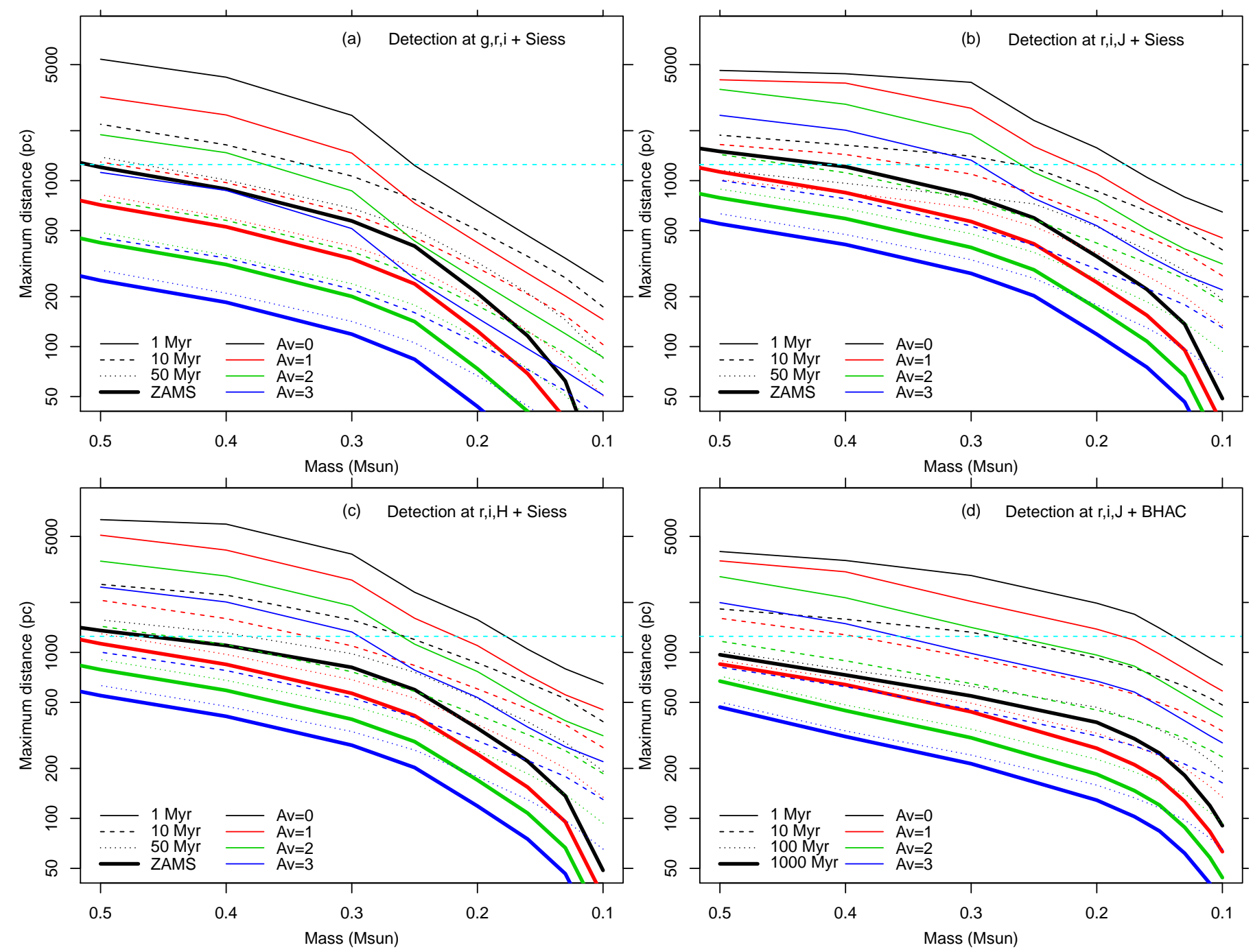

Fig. 3. Mass-distance-age (MDA) diagrams: maximum distances for simultaneous detection in three bands, using completeness limits from Fig. 2, and PMS evolutionary tracks from Siess et al. (2000) or BHAC, as indicated. The cyan horizontal dashed lines indicate the distance of the NGC 6530 cluster.

We adopted here the MYStIX catalog from Kuhn et al. (2013), including $2427 \mathrm{X}$-ray sources, but in practice restricted our considerations exclusively to the subset of 1756 sources with an optical, NIR, or Gaia counterpart, for the reasons explained above. The positional match between X-ray sources and the other catalogs was made within $2.5 \times \sigma_{\mathrm{Xpos}}$ summed in quadrature with 0.07 arcsec, where $\sigma_{\mathrm{Xpos}}$ is the X-ray position error, which varies from source to source because of counting statistics and PSF variations across the Chandra FOV, and 0.07 arcsec was adopted as a safety minimum match radius.

\section{Member selection}

\subsection{M-type stars}

We have shown in Paper I the effectiveness of the selection of PMS M-type stars based on multiband photometry. M stars are selected from three color-color diagrams (the $(r-i, g-r)$, $(r-i, r-J)$, and $(r-i, r-H)$ diagrams), where they lie along a locus which removes the $T_{\mathrm{eff}}-A_{V}$ degeneracy. $\mathrm{M}$ stars remain therefore well recognizable, unlike stars of other spectral types, at all values of extinction. Moreover, since PMS M stars are much brighter than main-sequence $M$ stars, they are observable up to much larger distances for given magnitude limits (as appropriate for a particular photometric dataset), and this makes a cluster rich in PMS M stars recognizable against a more diffuse field-star population of nearby M stars. For details, see Paper I. Quantitative predictions on detection limits for the dataset being studied here were made in the same way as in Paper I, on the basis of theoretical isochrones from Siess et al. (2000) and Baraffe et al. (2015; BHAC), the completeness limits for each photometric band, and knowledge of the reddening law (see Appendix B). For each value of age and extinction $A_{V}$ there is a minimum mass for a star to be detected in the photometric catalog, for a given distance of the cluster. Alternatively, for a given mass there is a maximum distance for detection for given age and extinction. These relations are graphically expressed by the mass-distance-age (MDA) diagrams, already used in Paper I, and reproduced for the case of NGC 6530 in Fig. 3. These diagrams differ subtly from those in Paper I, since the magnitude limits in the $J$ and $H$ bands are not the same, and since the reddening law was found here to differ slightly from that in Sco OB1 (see below). Moreover, we were here advantaged by the smaller distance of NGC 6530 compared to Sco OB1. The two diagrams on the right-hand side of Fig. 3 differ only in the theoretical tracks used, and give an idea of the model-related uncertainties 

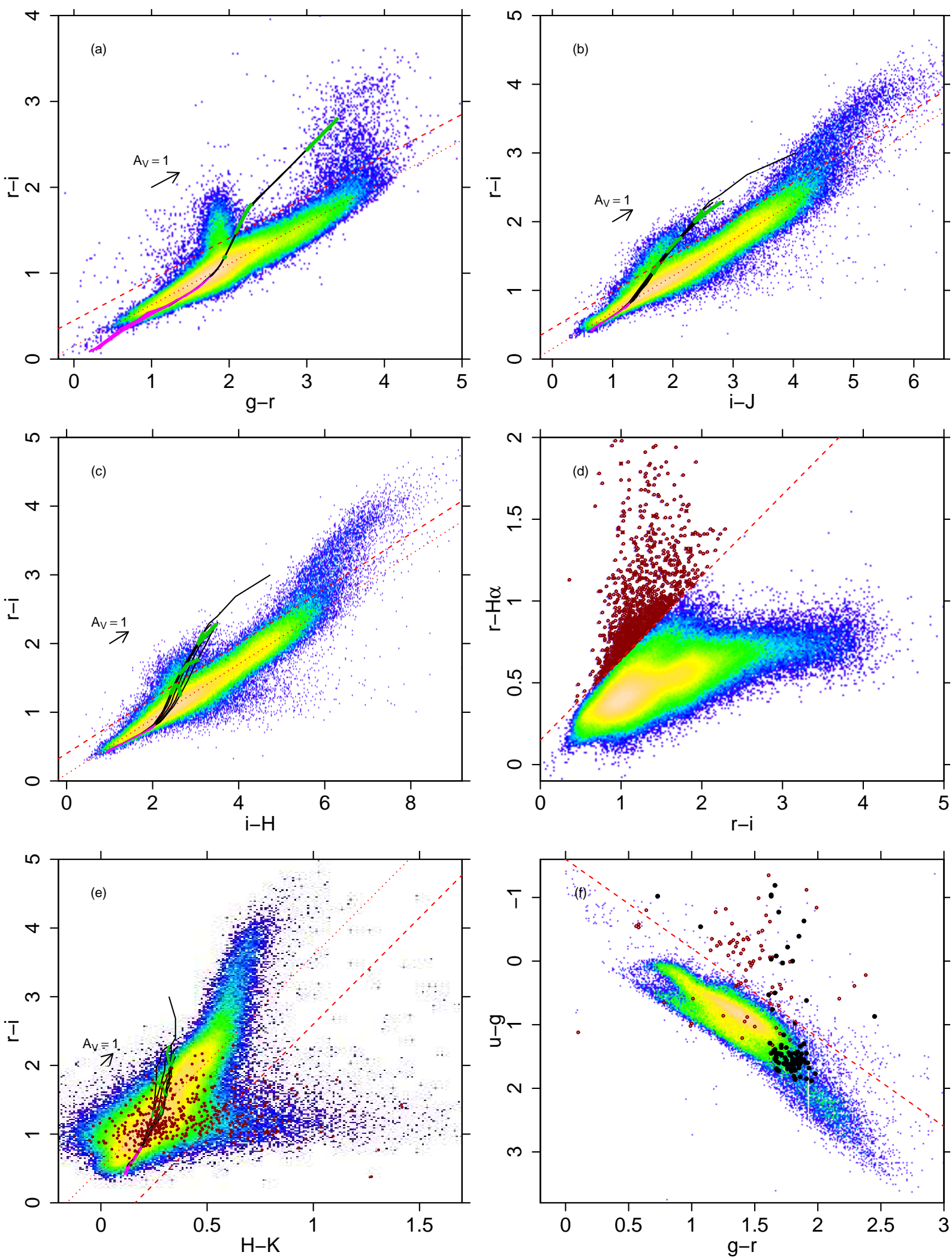

Fig. 4. Color-color diagrams used for selection of young stars. Each diagram is a two-dimensional histogram, with colors indicating the density of datapoints. Only data with errors less than $0.1 \mathrm{mag}$ on each pair of colors are shown. The dotted lines, parallel to the reddening vector (except for panel $d$ where no reddening vector is defined), describe the bulk of datapoints. Panels $a, b$ and $c$ : M stars are found above the dashed lines, parallel to the reddening vectors. Panel $d$ : strong $\mathrm{H} \alpha$-emission stars are found above the dashed line (dark-red points). Panel $e$ : IR-excess stars are found below the dashed line (dark-red points are the same as in panel $d)$. Panel $f:(g-r, u-g)$ color-color diagram for selection of UV-excess stars (above the dashed line). Black dots indicate M stars, while dark-red points are as in panel d. In panels $b, c, e$ : BHAC isochrones (evolutionary tracks) with black (green) lines for ages $1,10,50$ and $10000 \mathrm{Myr}$ (masses of $0.1,0.3$, and $0.5 M_{\odot}$ ), reddened as appropriate for NGC $6530\left(A_{V}=1.085\right)$. The location of stars with mass $M>1 M_{\odot}$ (all ages) is indicated with a magenta curve. In panel $a$ only, Siess et al. (2000) isochrones and tracks are used, the BHAC set being unavailable for the $g$ band. 
in the method; as is seen, these affect the details but not the basic pattern of predicted detections versus mass, distance, extinction, and age. As an example, the minimum detectable mass in $r, i, J$ at the NGC 6530 distance, $1 \mathrm{Myr}$ age, and $A_{V}=1$ $\left(A_{V}=2\right)$ is $0.22(0.26) M_{\odot}$ according to Siess models, while is $0.18(0.27) M_{\odot}$ according to BHAC. At $10 \mathrm{Myr}$ and $A_{V}=0$, Siess and BHAC predict minimum masses of respectively 0.26 and $0.285 M_{\odot}$.

The color-color diagrams used to select $\mathrm{M}$ stars from our optical-NIR catalog in the Lagoon nebula region are shown in the first three panels of Fig. 4. The large number of field stars (mostly background) contained in the catalog, spanning a very wide range of extinction, permits to define accurately the average reddening law (over a large distance range) in this sky direction. This is described by the dotted lines, fitted to the bulk of datapoints. The dashed lines separate $M$ stars (above them) from all other spectral types. While their slopes are fixed by the reddening vectors, intercepts were empirically determined as a compromise between maximum inclusion of M-type stars and minimum inclusion of reddened hotter stars. In all cases, the maximum mass they select is comprised between 0.3 and $0.5 M_{\odot}$ (thick green lines in the figure), the exact values being slightly agedependent. As also predicted by the model isochrones shown in the figure, the distribution of $\mathrm{M}$ stars does not run parallel to the reddening vector in each of the three $(r-i, g-r),(r-i, r-J)$, and $(r-i, r-H)$ diagrams. This makes also possible to determine rather accurate extinction values for individual $M$ stars, by back-projecting them onto the model tracks at zero extinction (except for the $(r-i, r-H)$ diagram, the model locus is not age-dependent). The general pattern in all three panels is very similar to that found in Sco OB1 (Paper I), with a lowextinction group of $\mathrm{M}$ stars well separated from another group of $\mathrm{M}$ stars at much higher extinction $\left(A_{V} \sim 8-10 \mathrm{mag}\right)$, which was argued in Paper I to be constituted by distant $\mathrm{M}$ giants. Below we present additional evidence (other than that provided in Paper I) that this is indeed the case. The NGC 6530 M-type members are therefore expected to belong to the low-reddening $M$ star groups (the MDA diagrams can also be used to confirm that we would be unable to detect any $1 \mathrm{Myr}$-old M stars in NGC 6530 with $A_{V}>5-6$, using the present catalog).

As in Paper I, we define three groups of $\mathrm{M}$ stars (i.e., above the dashed line in Fig. 4a), named after the respective diagrams, as

$M_{\text {gri }}:(r-i)>0.45+0.48(g-r)$,

and also $1.5<(g-r)<2.5$ to discriminate from high-reddening $\mathrm{M}$ giants.

$M_{\mathrm{riJ}}: \quad(r-i)>0.45+0.53(i-J)$,

and $1.2<(i-J)<3.0$.

$M_{\mathrm{riH}}:(r-i)>0.4+0.4(i-H)$,

and $2<(i-H)<4$. High-reddening $\mathrm{M}$ stars (giants) are defined using the same Eqs. (1)-(3), but above the limits $(g-r)>2.5$ $\left(g M_{\text {gri }}\right.$ stars $),(i-J)>3\left(g M_{\text {riJ }}\right)$, and $(i-H)>4\left(g M_{\mathrm{riH}}\right)$, respectively. The slopes used in Eq. (1)-(3) are the same as those of the reddening vectors.

The numbers of stars found in each group are $3298 M_{\text {gri }}$, $3547 M_{\text {riJ }}$, and $2970 M_{\text {riH }}$ stars; the latter two are largely overlapping, with 2631 stars in common, while there are only 696 stars in common between $M_{\text {gri }}$ and the combined $M_{\text {riJ }}$ plus $M_{\text {riH }}$ group. The total number of low-reddening $M$ stars is 6488 . For the high-reddening giants, the corresponding numbers are
$693 g M_{\text {gri }}, 2987 g M_{\text {rij }}$, and $2919 g M_{\text {riH }}$ stars, for a total number of 3620 stars; the overlap beween $g M_{\text {riJ }}$ and $g M_{\text {riH }}$ samples is nearly complete (2804 stars), while that between these two and the $g M_{\text {gri }}$ stars includes only 175 stars (the large extinction toward distant giants preventing $g$ band detection for most of them).

\subsection{Ho emission, NIR and UV excesses}

The $r-\mathrm{H} \alpha$ index from the VPHAS+ catalog was used to select CTTS from their strong $\mathrm{H} \alpha$ emission, using the diagram in Fig. 4d, in the same way as it was done in Paper I. We find in this way 1254 emission-line stars over the whole studied region, of which 150 also belonging to the low-reddening M-star sample. Using a similar, but more conservative selection method, Kalari et al. (2015) finds $235 \mathrm{H} \alpha$-emission stars inside a $50^{\prime} \times 30^{\prime}$ field in the Lagoon nebula region, smaller than the region considered here. Compared to Kalari et al. (2015), our selection is more inclusive of stars near the CTTS threshold.

Figure 4e shows an optical-NIR color-color diagram, useful to select Class II PMS stars with disk-related NIR excesses. As in Paper I (and already argued in Damiani et al. 2006b, 2017b, or Guarcello et al. 2007), this mixed optical-NIR diagram selects more objects than the $(J-H, H-K)$ diagram (not shown here), being sensitive even to weaker excesses in spite of requiring data at more bands. We find 2173 NIR-excess stars selected from Fig. 4e, of which only 73 in common with the low-reddening M-star sample, and 79 with the $\mathrm{H} \alpha$-emission sample. The total number of optical-NIR-excess objects is much larger than found in the analogous study by Damiani et al. (2006a - 333 sources), both because the NIR data used here are much deeper that the 2MASS data of the former study, and because of the much larger field studied here, despite that Damiani et al. (2006a) use several NIR-NIR and optical-NIR diagrams for the highest completeness while we here use only the $(r-i, H-K)$ diagram.

Finally, Fig. 4f shows the $(u-g, g-r)$ diagram, useful to select stars with an UV excess, which in the case of PMS stars is commonly attributed to the accretion spot where disk material hits the star surface. We find here 436 UV-excess stars, of which 17 among the $\mathrm{M}$ stars (the overall number of $\mathrm{M}$ stars with $u$ band data is anyway very small - black dots in Fig. 4f, 61 stars), and 75 among the $\mathrm{H} \alpha$-emission stars.

\subsection{Color-magnitude diagrams}

We show in Fig. 5 an optical and a NIR color-magnitude diagram (CMD) for all stars selected using the above methods as lowreddening $M$ stars, high-reddening $M$ stars, and $\mathrm{H} \alpha$-emission stars, respectively. Again, the overall distribution of datapoints is similar to that found in Sco OB1 (Paper I): neither the M stars nor the emission-line stars form sequences in the CMD, indicating that most of them are field objects. In particular, the bulk of $\mathrm{H} \alpha$-emission stars falls below the ZAMS at the cluster distance (and nominal extinction), suggesting they lie farther away. High-reddening $M$ stars are on average brighter than lowreddening M stars: this is particularly evident in the $(H, H-K)$ diagram in the right panel of Fig. 5 since these bands are less affected by extinction: this again supports the idea that they are giants, much brighter than $\mathrm{M}$ dwarfs despite their larger reddening and distance. The $(i, r-i)$ diagram shows a region with a reduced density of $\mathrm{M}$ stars, between the regions occupied by dwarfs and giants (indicated with a dashed line). A number of stars in the high-reddening sample (dark-green datapoints) fall below the dashed line, that is closer in the CMD to the dwarfs 

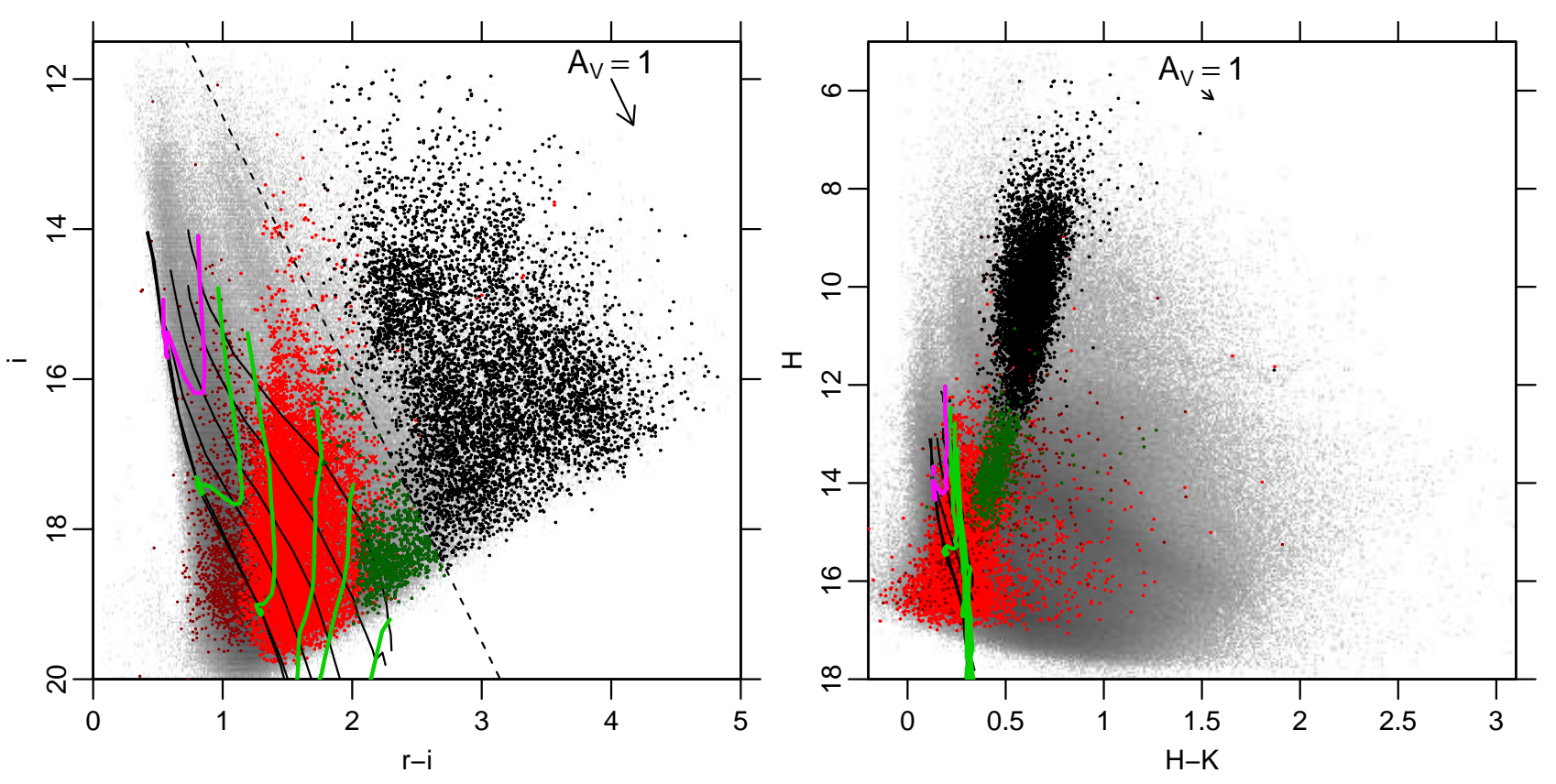

Fig. 5. Color-magnitude diagrams for all stars in the studied region (left: $(i, r-i)$; right: $(H, H-K))$. Only data with errors less than 0.1 mag on colors and magnitudes are shown. Tracks and isochrones (ages of 1, 3, 10,30, 100, 10000 Myr) as in Fig. 4, assuming a distance of $1250 \mathrm{pc}$ and extinction $A_{V}=1.085$. The magenta isochrones correspond to $1 M_{\odot}$ stars. Dark-red points indicate $\mathrm{H} \alpha$-emission stars, light-red dots low-reddening M stars, and black dots high-reddening M stars (giants). Dark-green dots are high-reddening M stars which by virtue of their position in the CMD are included in the low-reddening sample.

than to the giants. We have therefore reclassified this small group of stars, including them among the low-reddening M-star sample.

\subsection{Proper motion and parallax}

We also considered the data on proper motion $\left(\mu_{\alpha}, \mu_{\delta}\right)$ and parallax $(\pi)$ provided in the Gaia DR2 catalog (Luri et al. 2018), as a further membership criterion. The NGC 6530 cluster is not immediately visible in the proper-motion plane using the Gaia data, its motion being not dramatically different from that of the much more numerous field stars in its surroundings. The same holds for the parallax-proper motion plane (either along RA or Dec). We have therefore used a differential approach, by defining an initial "cluster" region, and a comparison "reference" region where we have no indications of the possible presence of cluster members. The cluster region was chosen from the stars fulfilling any of the $\mathrm{H} \alpha$, NIR, and UV selection criteria (named collectively the candidate CTTS stars). The (smoothed) spatial density of CTTS is shown in Fig. 6. The enhancement near the center, corresponding to NGC 6530 is evident. A density gradient from SE toward NW (i.e., toward the Galactic plane) is also clearly seen, and $\mathrm{H} \alpha$ or NIR-excess stars near the plane are neither clustered nor related to NGC 6530. The enhancement in the SE corner corresponds to the globular cluster NGC 6544. From the figure, we set the initial cluster region, enclosing nearly all of its possible candidate members, as that inside the red rectangle, and the reference region as that between the red and the blue rectangles.

The density of datapoints in the proper-motion plane, including only stars in the cluster region, is shown in Fig. 7a: superimposed to the distribution of the field stars (the majority of datapoints, in a nearly-elliptical distribution centered on $\mu_{\alpha} \sim$

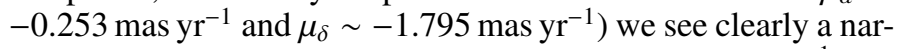

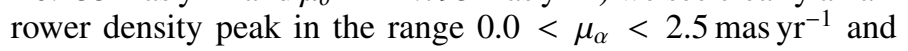

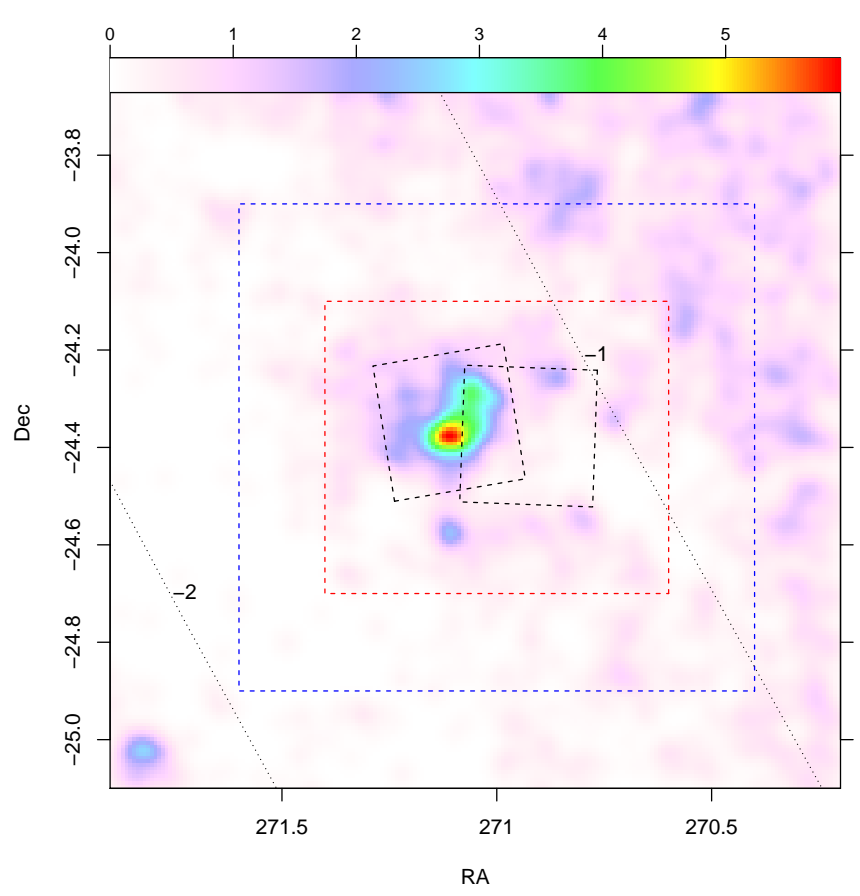

Fig. 6. Smoothed density map of all stars with either $\mathrm{H} \alpha$ emission, NIR or UV excesses. The smaller black squares are the Chandra FOVs as in Fig. 1. The red dashed rectangle is a provisional cluster region, while the outer blue rectangle indicates the surrounding reference field-star region. The oblique dotted lines indicate constant Galactic latitude $b$, as labeled. The enhancement in the lower-left corner corresponds to the globular cluster NGC 6544. Labels in the top-axis colorbar indicate density in units of sources per square arcmin.

$-3.5<\mu_{\delta}<-1.0$ mas $\mathrm{yr}^{-1}$. The density distribution in the reference field is shown in the same Fig. 7a with the dashed contours, which only indicate the presence of the field-star component, as 

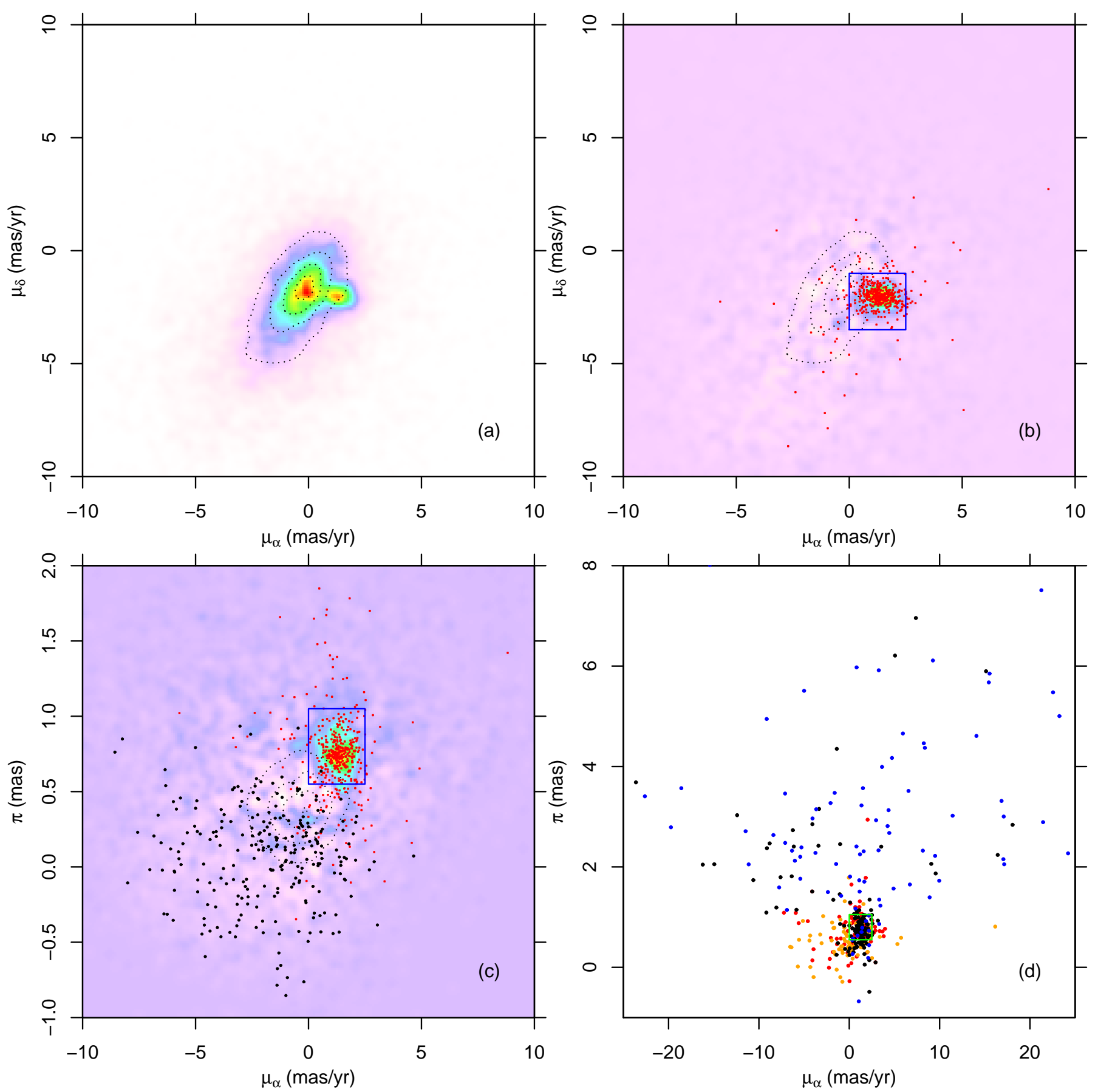

Fig. 7. Panel $a$ : distribution in proper-motion plane of all Gaia sources falling in the cluster region defined in Fig. 6, and having errors on $\mu_{\alpha}$ and

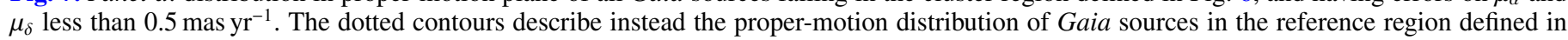
Fig. 6, excluding those in the cluster region. Panel b: map of density difference between Gaia sources in cluster and reference regions, scaled by respective total sources numbers, in proper-motion plane. Red dots are NGC 6530 X-ray sources. The blue rectangle indicate the cluster boundaries in the proper-motion plane. Contours are the same as in panel a. Panel c: map of density difference between Gaia sources in cluster and reference regions, scaled by respective total sources numbers, in $\mu_{\alpha}$-parallax $(\pi)$ plane. The maximum parallax error for inclusion is 0.3 mas. Red dots are NGC 6530 X-ray sources. Black dots are candidate M giants. The blue rectangle indicate the cluster boundaries in the $\mu_{\alpha}$-parallax plane. Contours describe the distribution of sources in the reference region. Panel $d$ : wider-scale view of the $\mu_{\alpha}-\pi$ plane of panel c. The green rectangle is the same as the blue rectangle in panel c. Black (blue) dots indicate low-reddening M stars in the cluster (reference) region, while red (orange) dots indicate CTTSs in the cluster (reference) region.

expected. Only sources with errors on both $\mu_{\alpha}$ and $\mu_{\delta}$ less than

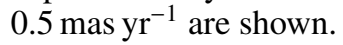

In order to better isolate the cluster in the proper-motion plane we normalized both the cluster and reference distributions to unity (to account for the difference in the respective number of stars), and plotted their difference in Fig. 7b. This fluctuates around zero, except for the cluster stars which form a welldefined density peak (outlined with a blue rectangle). It should be clear that the normalization procedure is only an approximation, both since it does not take into account the expected larger 
density of stars in the cluster region because of the cluster contribution, and since the non-uniform spatial obscuration does not guarantee that the properties of the field stars in the two regions compensate exactly. The map of Fig. 7b, moreover, has by construction zero average, so that the positive cluster peak must be balanced by a sparser distribution of negative values (the bluest ones). Nevertheless, this representation serves its main purpose very well, the cluster peak being evidently well defined. In the same panel, we show all X-ray detections with a match in the Gaia catalog: a negligibly small percentage of them lies outside the blue rectangle (the "cluster box"), well compatible with either spurious X-ray-Gaia associations or field X-ray sources. The bulk of X-ray detections, which are good candidate cluster members, confirm that the excess density peak in the propermotion plane does indeed correspond to NGC 6530.

The same differential procedure was applied to the $\left(\mu_{\alpha}, \pi\right)$ plane, with the result shown in Fig. 7c: there is a well-defined peak in the differential density map for $0.55<\pi<1.05$ mas (blue rectangle), with X-ray detections confirming its coincidence with the cluster. Only sources with error on $\pi$ less than 0.3 mas are shown, and proper-motion error less than

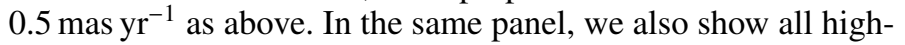
reddening $\mathrm{M}$ stars as black dots: virtually none of them lies inside the cluster box, nor do they form any cluster in this plane; their parallaxes are on average much smaller (often consistent with zero ${ }^{1}$ ) than for NGC 6530 stars, which confirms that they lie at much larger distances, and we are correct in our arguments that they must be luminous and distant giants.

Finally, Fig. 7d shows the same density difference as panel $c$, but uses wider boundaries in both axes, to show not only the lowreddening $M$ stars in the cluster region (most of them inside the cluster box, in agreement with our arguments on M-type member selection), but also the sparse population of field M (dwarf) stars in the reference region (blue dots), which as expected from our earlier arguments are found scattered over a large range of $\mu_{\alpha}$ values, and at definitely larger $\pi$ values (smaller distances) than cluster stars: the vast majority of M-type dwarfs are too faint to be detected in our dataset at the NGC 6530 distance, as predicted by Fig. 3. Panel d also shows the distribution in the $\left(\mu_{\alpha}, \pi\right)$ plane of the candidate CTTS from the cluster and reference regions: while those in the cluster region are mostly found inside the cluster box, those in the reference region are scattered more widely, and often at smaller $\pi$, indicating that they constitute an unrelated population of emission-line or NIR-excess objects (so that the CTTS designation may only be meaningful for the objects inside the cluster region).

Therefore, we consider as Gaia-selected candidate members all sources with $0.55<\pi<1.05$ mas, $0.0<\mu_{\alpha}<2.5$ mas yr $^{-1}$,

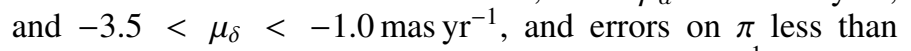
0.3 mas, and errors on $\mu_{\alpha}$ and $\mu_{\delta}$ less than 0.5 mas yr$^{-1}$.

\subsection{Refining photometric selection}

By combining several of the previous member selection methods, we were able to determine a highly reliable subsample of cluster members, from which we in turn derived other properties of the whole population, and additional constraints. In particular, NGC 6530 members occupy a well-defined portion of the optical CMD, as is shown in Fig. 8 where we only show members selected using Gaia plus one of the other methods. The complementarity of the methods is evident from the fact that the

\footnotetext{
1 See Luri et al. (2018) regarding the meaning of negative $\pi$ measurements.
}

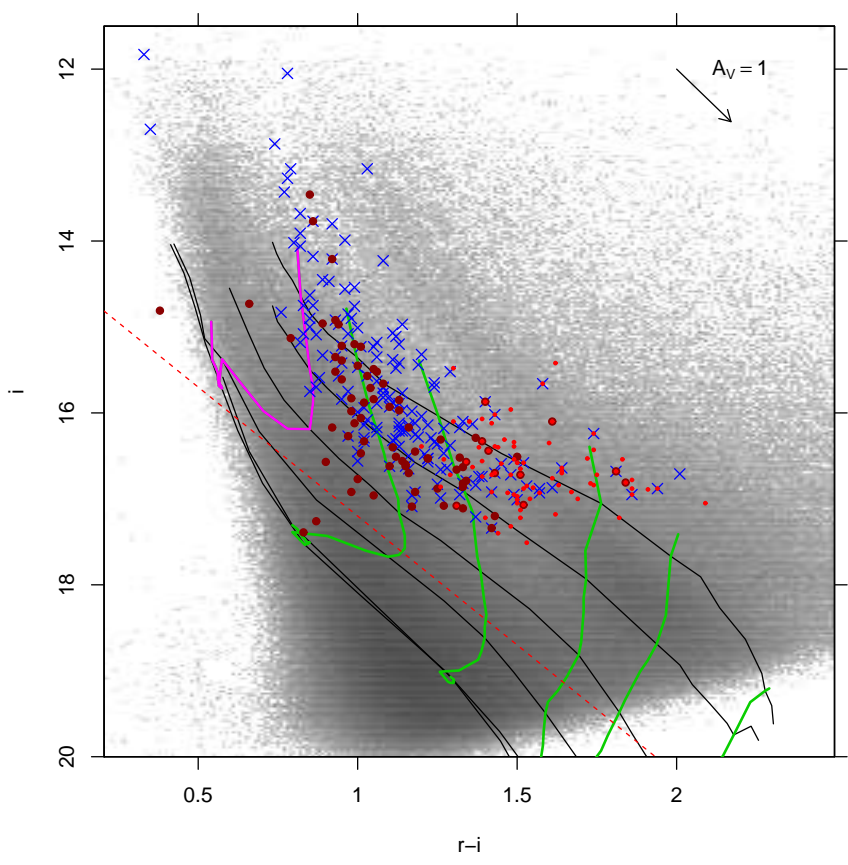

Fig. 8. $(i, r-i)$ color-magnitude diagram of $\mathrm{M}$ stars (red dots), X-ray sources (blue crosses), and CTTSs (dark-red points) which are also selected using Gaia proper motions and parallaxes. Background grayscale image: same CMD as in Fig. 5a. Evolutionary tracks, isochrones and reddening vector as in the same figure. The red dashed line (roughly corresponding to a $25 \mathrm{Myr}$ isochrone) delimits the CMD region occupied by X-ray and M-type members.

respective samples tend to populate different parts of the CMD, with limited overlap between them. As expected, X-ray detections become rarer toward the lowest masses because of sensitivity limitations and X-ray luminosity scaling with mass (e.g., Damiani et al. 2006b and references therein), which emphasizes the importance of a method for selection of the cluster M stars. On the other hand, CTTSs are scattered across the entire mass range, and in some cases at apparent ages older than the rest of the cluster members: this fact was already noticed both in NGC 6530 (Damiani et al. 2006a) and in other young clusters (NGC 6611, Guarcello et al. 2007; NGC 6231, Damiani et al. 2017b) and is probably unrelated to the stellar ages. We observe that no X-ray or M-type stars are confirmed as members using Gaia below a certain limit, shown as a red dashed line in the figure, which approximately corresponds to a $25 \mathrm{Myr}$ isochrone. Stars below that limit in the CMD are thus very unlikely to belong to the cluster, unless they show strong spectral peculiarities (in fact, only two CTTSs fall below it in Fig. 8). We therefore applied this additional constraint to our M-star selection, which removed 1083 main-sequence $M$ stars at distances close to NGC 6530. The same constraint was applied to CTTSs, also considering that the CTTSs falling below the red dashed line in Fig. 8 show no spatial clustering. The number of CTTSs reduced therefore to 513 stars. All NGC 6530 candidate members selected using the various methods described are listed in Table 1.

\section{Results}

\subsection{Distance}

As can be seen from Fig. 7c the $\pi$ distribution of the cluster stars is rather wide: this was not unexpected and consistent with the 

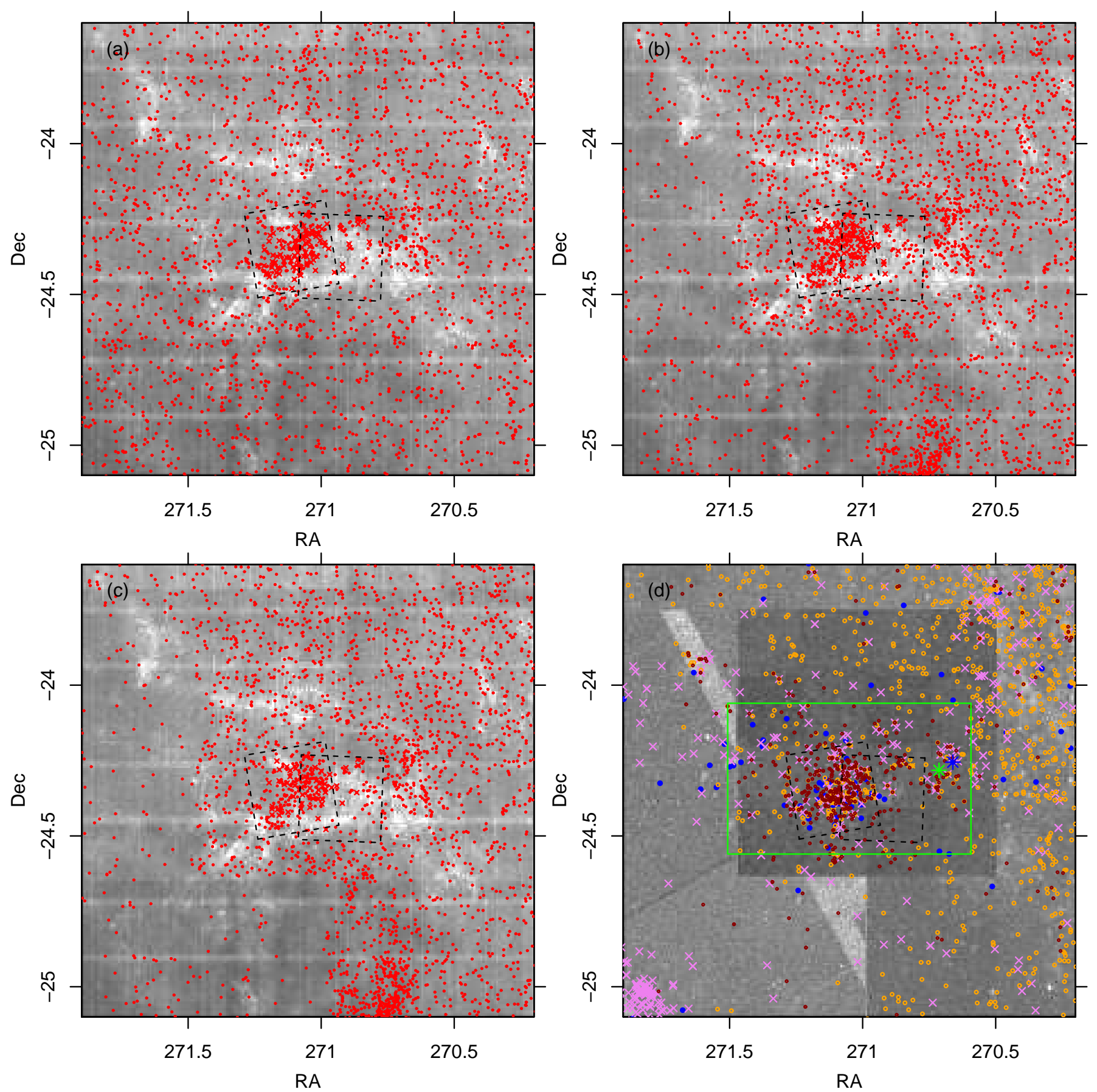

Fig. 9. Spatial distributions of star subsamples in the studied region. The background image is a two-dimensional density histogram of stars in VPHAS+ DR2 (and sources in our NIR catalog in the bottom right panel). The dashed squares are the Chandra FOVs. Top left: $M_{\text {gri }}$ stars (red) selected from Fig. 4a. Top right: $M_{\mathrm{riJ}}$ stars (red) from Fig. 4b. Bottom left: $M_{\mathrm{riH}}$ stars (red) from Fig. 4c. Bottom right: dark-red points, orange circles, and larger purple crosses are stars with $\mathrm{H} \alpha$ emission, optical-NIR excesses, and UV-excesses, respectively, as in Fig. 4; big blue dots are O or B stars from SIMBAD. The green rectangle is the field studied by Kalari et al. (2015). The green and blue asterisks are the stars 7 Sgr (F2II-III) and HD $164536(\mathrm{O} 7.5 \mathrm{~V})$, respectively.

current precision of parallax measurements in Gaia DR2, also considering that the majority of cluster members are faint stars and precision on $\pi$ degrades with magnitude. The actual distribution of distances expected among cluster members cannot justify a range in $\pi$ from 0.55 to 1.05 (nominally, $950-1820 \mathrm{pc}$ ). The Gaia data, therefore, do not resolve the cluster along the line of sight, and by consequence we may only derive an average value for the cluster distance, using a suitable average of $\pi$ values of its best candidate members. Considering the subsample of very good candidate members which are both X-ray detected and falling inside the cluster box in both proper motion and parallax (292 stars), their median parallax is 0.75015 mas. The crucial issue in converting parallaxes to distances is that the error distribution on the former is symmetric and Gaussian, while that on distances computed simply as $d=1000 / \pi$ is asymmetric, and the amount of asymmetry depends on relative error on parallax, $\Delta \pi / \pi$. If $\Delta \pi / \pi$ is sufficiently small (precise $\pi$ measurements), say $\Delta \pi / \pi<0.1$, the asymmetry introduced by inversion 


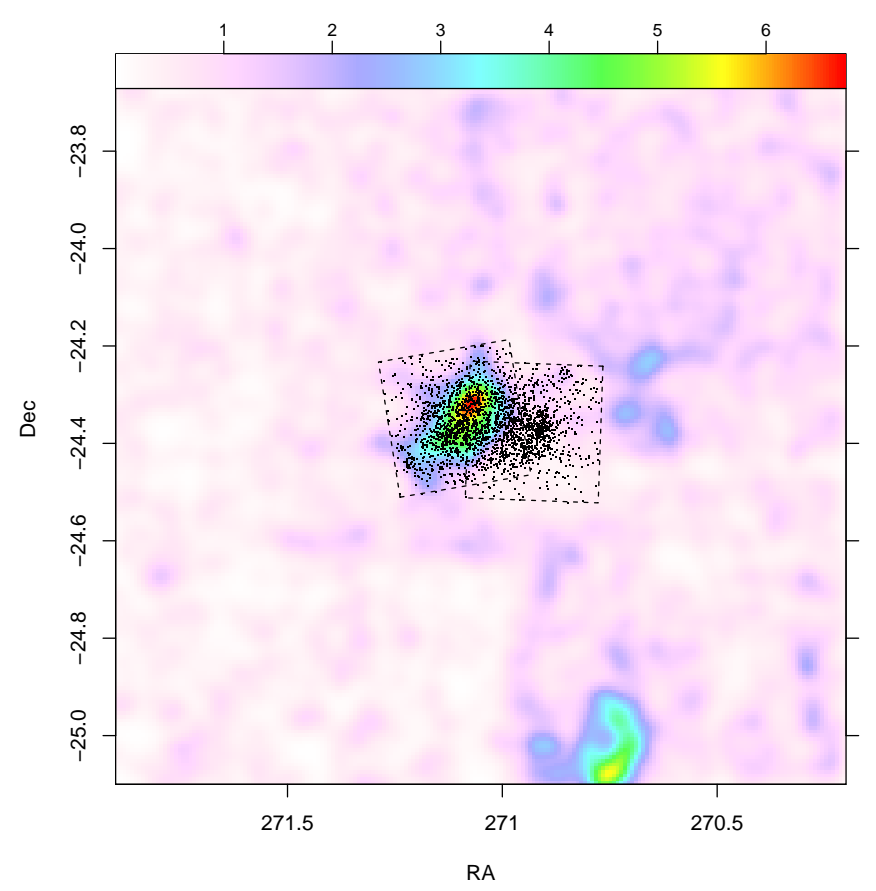

Fig. 10. Smoothed spatial distribution of all low-reddening $M$ stars. The black dashed regions are the Chandra FOVs, and dots are X-ray sources. Top-axis colorbar as in Fig. 6.

is negligible, and $d=1000 / \pi$ is a reliable distance estimate (see Luri et al. 2018; Bailer-Jones et al. 2018). As recommended by these authors, modeling should be done as far as possible directly using parallaxes, and conversion to distances should be the last operation performed; parallax errors being Gaussian, error propagation is much more accurate using parallaxes as well. Therefore, we computed first the mean parallax of NGC 6530 members and its error, and then converted them to distance and its error. The error-weighted mean parallax for the best candidate members is 0.75449 mas, very close to the median, with an error on the mean (not the standard deviation of datapoints) equal to 0.00412 mas. Therefore, for the cluster as a whole we obtain $\Delta \pi / \pi=0.0055$, definitely small enough for direct distance computation using $d=1000 / \pi$. The corresponding distances are $1333 \mathrm{pc}$ for the median parallax and $1325_{-7.2}^{+7.3} \mathrm{pc}$ for the weighted mean, the distance error being slightly asymmetric whereas the error on $\pi$ is symmetric (Luri et al. 2018). While very precise, this distance determination is likely not accurate: Arenou et al. (2018) report that Gaia DR2 parallaxes show systematic residuals (compared to reference values) of $\sim-0.065$ mas, that is the Gaia distances seem to be overestimated, by $\sim 113 \mathrm{pc}$ in the case of NGC 6530. Were it not for this effect, the Gaia data would locate the cluster $75 \mathrm{pc}$ more distant than found by Prisinzano et al. $(2005 ; 1250 \mathrm{pc})$, but taking into account the above Gaia systematic error the new Gaia distance is well in agreement with the previous value.

\subsection{Spatial morphology}

We discuss here the spatial distribution of candidate NGC 6530 members according to the different membership criteria from the previous Section. Figures 9 -a,b,c show the distributions of $M_{\text {gri }}$, $M_{\text {riJ }}, M_{\text {riH }}$ stars, respectively. Figure 9d shows instead the spatial distributions of CTTS candidates (i.e., $\mathrm{H} \alpha$-emission, NIR- and UV-excess stars). The analysis of the $M_{\text {gri }}$ stars benefits from the uniform catalog coverage across the entire region studied, and shows clearly a clumpy distribution near the cluster center (with the largest concentration in the cluster core region, inside the eastern Chandra FOV), and a uniform distribution in the diffuse field. Stars in the latter group are field M dwarfs, as also suggested in Sect. 3.4, found at smaller distances than the NGC 6530 cluster. The effect of mixing different NIR catalogs is clear in panels b, c, and d, with a sharp density drop at their interface, because the filtering applied to the VVV data made this catalog less complete than the King-UKIDSS catalog in the central regions. Within these limitations, the pattern of $M_{\mathrm{riJ}}$ and $M_{\mathrm{riH}}$ stars in panels $\mathrm{b}$ and $\mathrm{c}$ is consistent with that derived from panel a, except for a conspicuous excess near $(\mathrm{RA}, \mathrm{Dec})=(270.8,-25)$. Tracing back the position of these "excess" stars in the defining color-color diagrams $(r-i, i-J)$ or $(r-i, i-H)$ we checked that they lie just above the threshold, at moderately large reddenings. The most plausible explanation for the absence of a similar clustering among the $M_{\text {gri }}$ stars in panel $a$ is that along that line of sight the NIR reddening law is slightly steeper than elsewhere, in the sense that stars deviate above the main trends in the relevant color-color diagrams. There is no indication of any type that a real cluster of stars exists around that position, from either CTTS diagnostics or Gaia data. The possibility of a nonuniform reddening law should be kept in mind before interpreting too naively the results from the M-star selection method. Under this respect, the three different ways of selecting $\mathrm{M}$ stars are useful for a mutual plausibility check, as in this case.

The distribution of $M$ stars in the cluster (inside the Chandra FOVs and in their immediate neighborhood) is remarkably consistent between the $M_{\text {gri }}, M_{\text {riJ }}$, and $M_{\text {riH }}$ subsamples (panels a, b, and c). It also agrees very well with the distributions of $\mathrm{H} \alpha$-emission stars, NIR- and UV-excess sources in panel d. Just to the west of the rightmost Chandra FOV, the M-star selection finds small groups of candidate members, which are also seen in panel d: these are among the westernmost stars for which there is convincing evidence of membership to NGC 6530. Kalari et al. (2015) also remarked that these stars are good candidate members on the basis of $\mathrm{H} \alpha$ and UV excess, and their neighborhood should be more carefully studied (the field examined in that work is outlined in panel d). In their vicinity lie the stars 7 Sgr (F2II-III) and HD 164536 $(\mathrm{O} 7.5 \mathrm{~V})$, also indicated in panel $\mathrm{d}$, which were found probably responsible for the local expansion of the ionized nebular gas by Damiani et al. (2017a).

Near the southern edge of the Kalari et al. (2015) field, approximately 15 arcmin south of the cluster core, a small group of $\mathrm{H} \alpha$ emission stars is found in panel d, whose membership to NGC 6530 needs to be considered carefully. However, we do not find them among the M-type candidates, nor among the Gaia candidates (see below), so it becomes more likely that they are an unrelated group of emission-line stars. As already shown in Fig. 6, NGC 6530 lies $\sim 1.5^{\circ}$ below the Galactic plane, which lies to the northwest of our studied field. The increase in the density of NIR-excess objects toward the northwest, as seen also in panel $\mathrm{d}$, is therefore ascribable to a diffuse galactic population, not connected to NGC 6530 .

To the southeast of the NGC 6530 core, partially inside the eastern Chandra FOV, a group of candidate members is found (from both the M-star criteria and the various CTTS criteria), which follows the profile of the local bright-rimmed cloud, in which the massive protostar M8-East IR (Wright et al. 1977) is embedded. A group of hard (probably embedded) X-ray sources close to that bright rim was also noted in the first Chandra X-ray study by Damiani et al. (2004). 

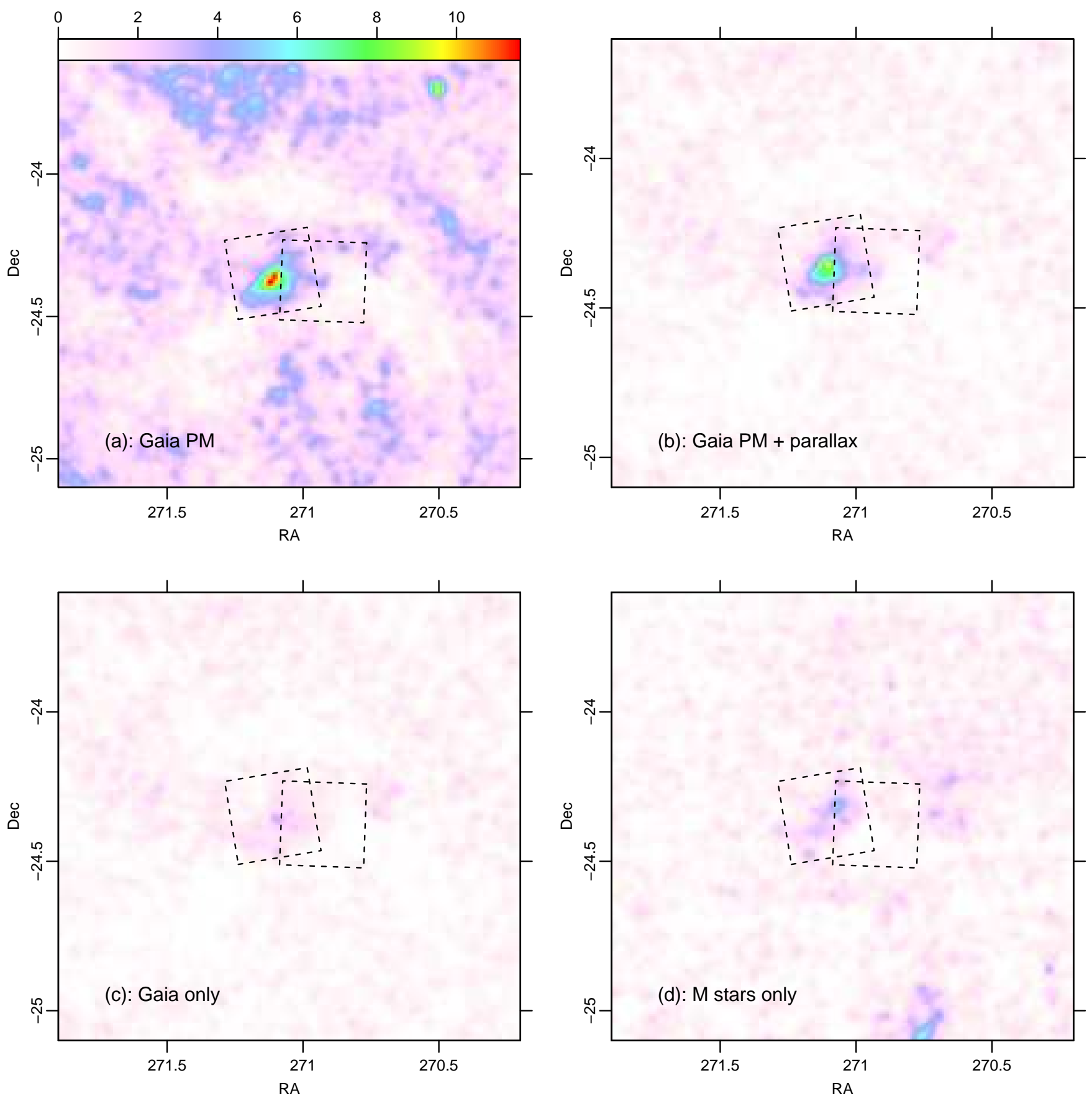

Fig. 11. Spatial distributions of subsamples selected according to various criteria. Dashed squares are the Chandra FOVs. The color scale is the same for all panels. Panel a: all Gaia sources selected from proper motion alone, falling inside the rectangle in Fig. 7b. and errors on $\mu_{\alpha}$ and $\mu_{\alpha}$ less than 0.5. Top-axis colorbar as in Fig. 6. Panel b: Gaia sources selected from proper motion and parallax, falling inside both rectangles of Fig. 7b and c. Panel c: Gaia sources as in panel b, but missed by the X-ray, CTTS, and M-star selection methods. Panel d: M stars missed by the Gaia, $\mathrm{X}$-ray, and CTTS selection methods.

Near the Hourglass nebula (center of rightmost Chandra FOV) no M-type members are found: this is a bias, caused by the lack of data in the VPHAS + catalog from that neighborhood in the $r$ band (probably as a result of the very bright $\mathrm{H} \alpha$ nebulosity), which is required by all three M-star selection criteria.

Figure 10 is a slightly smoothed map of the spatial distribution of all low-reddening M-type stars, compared to that of individual Chandra X-ray sources. We again remark that the rightmost Chandra FOV is much deeper than the other one, so that X-ray detections there are overrepresented. In the same FOV, the lack of M-type stars just discussed is evident. In the cluster core (left) FOV, instead, we find a very good agreement between the distribution of X-ray sources and that of M stars, indicating once again that the vast majority of both samples consists of very good candidate members of NGC 6530. The southeastern region (partially inside the lower-left corner of the left Chandra FOV) has a distinct morphology, slightly separated from cluster core and elongated along the bright rim (see, e.g., the VPHAS+ $\mathrm{H} \alpha$ images in Kalari et al. 2015 or Damiani et al. 2017a); the M-star sample shows that the member distribution along the 
rim continues even slightly outside the Chandra FOV to the east.

To the west of the Chandra FOVs, three groups of M stars are clearly found, of which two associated with the stars $7 \mathrm{Sgr}$ and HD 164536 as discussed above, and a third (rightmost) one not associated with anything obvious. In the next subsections we discuss that this latter subgroup is likely not associated with the cluster.

Next, we compared the spatial distributions of candidate members according to different combinations of selection criteria, to study the respective performances. For example, Fig. 11a shows the result of selecting stars exclusively using Gaia proper motions (ignoring parallaxes) in the cluster box of Fig. 7: this is not very effective, the NGC 6530 cluster being still recognizable, but accompanied by several other local density enhancements. After adding the parallax constrain (panel b), instead, NGC 6530 becomes much better defined, together with its small peripheral subgroups of likely members mentioned above, such as those in the southeast, and two out of three to the west of the Chandra FOVs. Since all these structures were already found by other selection criteria, the next question is whether the Gaia data do actually add any new members to the list assembled from M stars, CTTSs, and X-rays: panel c shows candidate members exclusive to the Gaia sample, and it does indeed prove that Gaia still adds some members to the pre-existing list, with a comparable distribution in space (including subgroups). The complementary question was if the M-star methods is of any usefulness, once we have assembled the Gaia, X-ray, and CTTS sample: panel $\mathrm{d}$ shows that there are still $\mathrm{M}$ stars which failed all other selection criteria, clearly with the same spatial shape of the cluster. We conclude that all four methods we used here are highly complementary to one another, each one being most effective in a particular region of the (large) parameter space covered by the NGC 6530 member stars. Therefore, the NGC 6530 member list obtained from the combination of all of these criteria is definitely more complete than those obtained from the individual criteria, or even by omitting just one of them. The exact completeness limit at the low-mass end depends on age and extinction, as can be seen from Fig. 3. We should remark that this holds in this particular cluster, whose members span a wide range of masses, extinction values, ages, and other peculiarities typical of the PMS phase; we expect that a similar situation holds for other very young clusters, but not for older clusters containing only main-sequence stars (e.g., the Pleiades) or giants.

Finally, the complete member list obtained from combining all our selection methods yields the spatial distribution shown in Fig. 12. The comparison between the cluster-related density enhancement with the fluctuations in the surrounding field-star density suggests strongly that all possible cluster members are contained within the rectangular regions shown in red: this is our final "cluster" region. There is no diffuse population of members outside of it. The secondary peak at the Hourglass, where optical data are highly incomplete because of the very bright nebulosity, is clearly defined thanks to the X-ray data. Since there is no comparably bright nebulosity elsewhere in the studied region, it is unlikely that the optical data (including Gaia) would miss a substantial percentage of stars in any subregion other than the Hourglass. The southeastern M8-East region (along the bright rim) is also identifiable in Fig. 12 with its elongated shape, as are also the minor condensations to the northwest.

\subsection{Extinction}

One of the very useful properties of $M$ stars is that their position on the color-color diagrams of Figs. 4-a,b,c is not

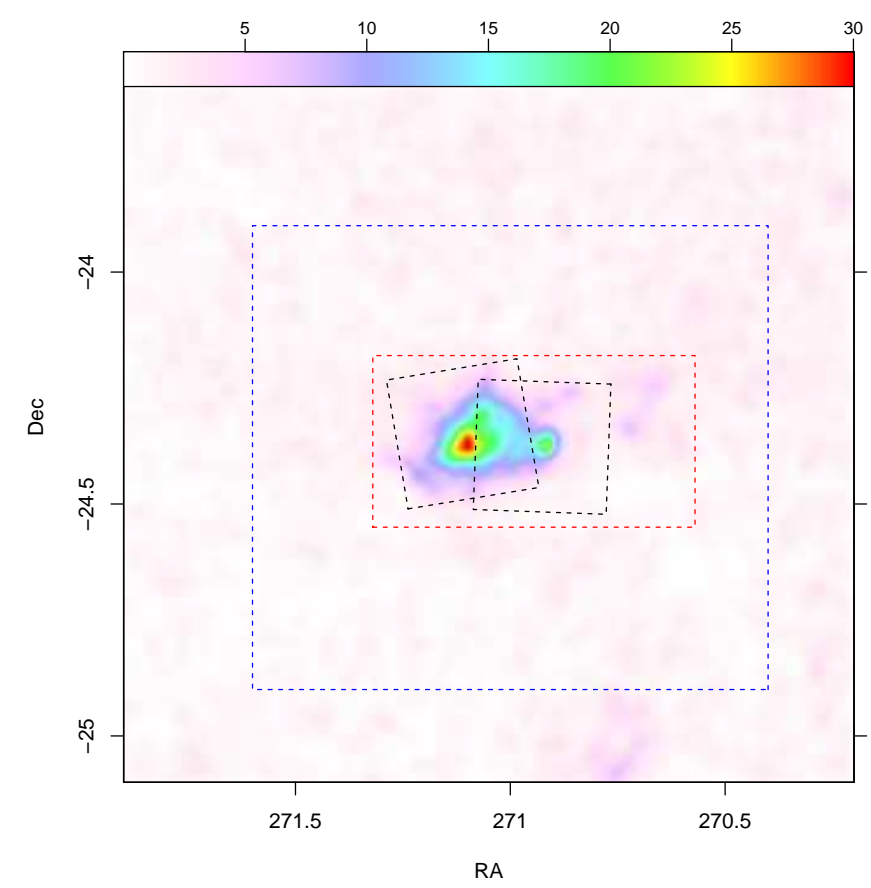

Fig. 12. Spatial map of candidate members selected by any method: Gaia proper motion and parallax, CTTS diagnostics, M-type selection (but brighter than the red line in Fig. 8), and X-rays. The black squares are the Chandra FOVs. The inner (red) rectangle is the final "cluster" region, while the outer (blue) one is the same reference region as in Fig. 6.

degenerate between $T_{\text {eff }}$ and extinction $A_{V}$. Therefore, adopting a zero-extinction locus (from a theoretical isochrone) and a reddening vector we were able to determine $A_{V}$ for each individual $\mathrm{M}$ star. In particular, we used $A_{V}$ as determined from the $(r-i, i-J)$ diagram, which yielded the largest number of candidates. In this way we derived $A_{V}$ for $3615 \mathrm{M}$ stars in the entire region, of which 627 in the cluster region (55\% of all cluster $\mathrm{M}$ stars). We therefore show the spatial distributions of $\mathrm{M}$ stars in four different $A_{V}$ ranges in Fig. 13. The literature $A_{V}$ for NGC 6530 is 1.08 . In panel a, M stars with $0<A_{V}<0.8$ do not show any clustering at the cluster position, and it seems legitimate to conclude that nearly all stars in this extinction range are foreground field stars. Two of the subgroups to the west of the Chandra FOVs are clearly found in this extinction range, however.

In all higher extinction ranges, instead, the cluster is very evident, but the higher-extinction members are distributed differently from the low-extinction members. The third (westernmost) subgroup outside the Chandra FOVs, mentioned above, disappears at higher extinction, while the two closer ones (associated with $7 \mathrm{Sgr}$ and HD 164536) are still visible, and therefore partially obscured by the M8 dark nebulosity. Not surprisingly, the highest extinction M-type members (panel d) are mostly found along the southern bright rim mentioned above, and partially embedded in its dust. Less expected, instead, is the presence of high-extinction members near the cluster core, where most stars have low extinction. Stars in that direction, therefore, are distributed along the line of sight at different distances, even until fairly deep within the dust cloud. In panel d, a bright spot near the top of the rightmost Chandra FOV coincides with a compact group of Chandra X-ray sources, also visible in Fig. 10, which therefore constitute likely a small group of very young, embedded low-mass stars. There is no indication of a diffuse population 

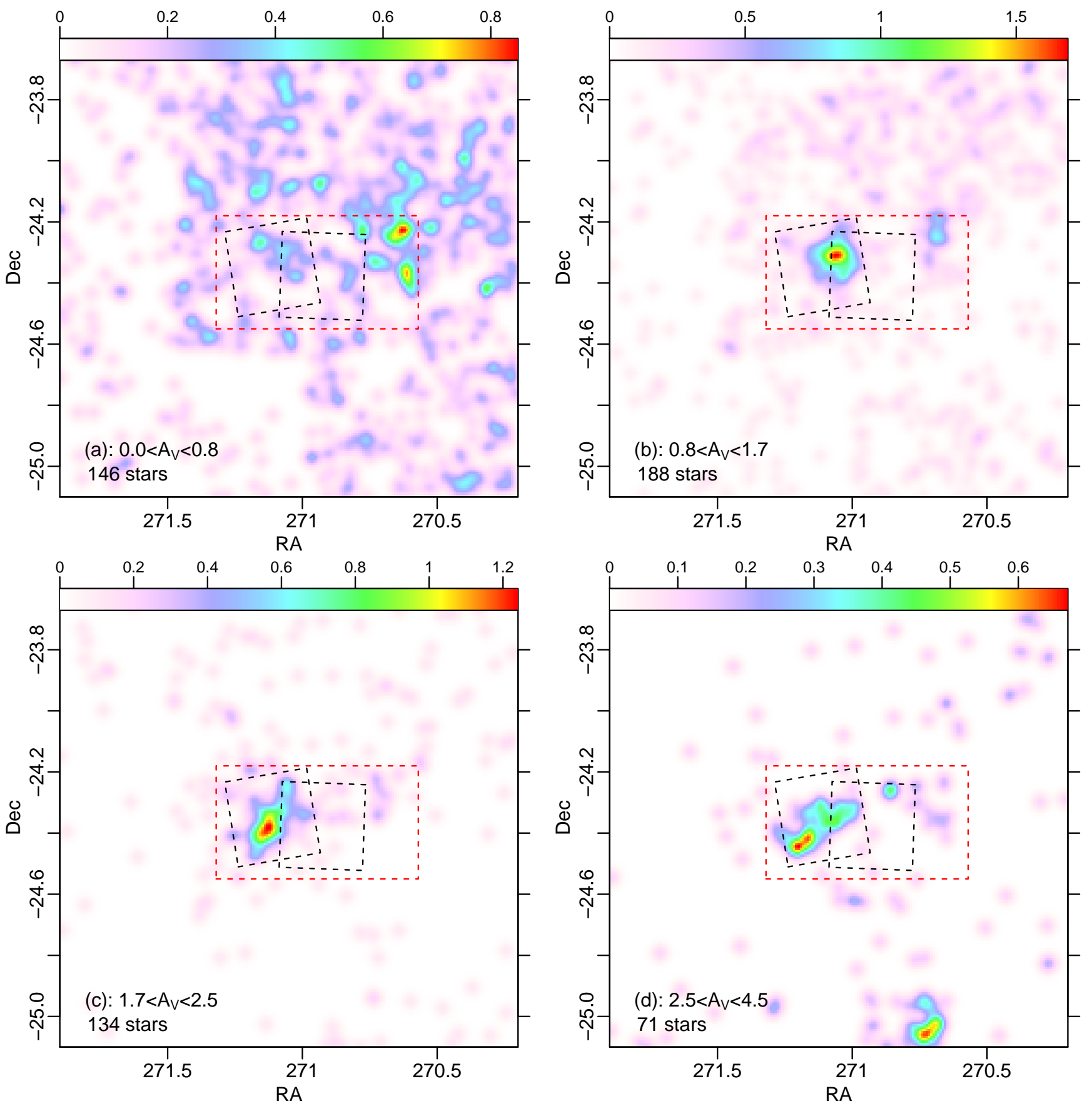

Fig. 13. Maps of spatial distribution of low-reddening M stars, for different $A_{V}$ ranges. For $A_{V}>4.5 \mathrm{mag}$ (not shown), there is no sign of clustering around the NGC 6530 position. Dashed squares are the Chandra FOVs. The dashed red rectangle is the cluster region as in Fig. 12. The number of $\mathrm{M}$ stars in this region is indicated for each $A_{V}$ range.

of NGC 6530 members, in any $A_{V}$ range. Overall, the largest number of clustered $\mathrm{M}$ stars falls within the $A_{V}$ range 0.8-2.4, with a tail ( $20 \%$ of the stars) at higher $A_{V}$.

While interpreting this type of maps, however, it should be kept in mind that larger extinction values correspond to decreased completeness in the sample. The extinction corrected CMD of M stars in the cluster region is shown in Fig. 14 for stars in four extinction ranges, where we also show the completeness limit at the end of each range (i.e., we show conservative estimates for the limits). Stars in the lowest extinction range are not expected to be members, as just discussed. At higher extinction, the lowest-mass stars can only be present in the lowest $A_{V}$ range, and significantly less present for increased extinction. The same holds for the oldest cluster members. This effect would introduce a spurious correlation between age and extinction if not properly accounted for, as that one would naively infer from the same diagram, by noticing that blue dots (less extincted stars) lie systematically below the red dots (highest-extinction stars). The existence of still higher-extinction NGC 6530 members, which are missing in this and other studies based on optical data, is 


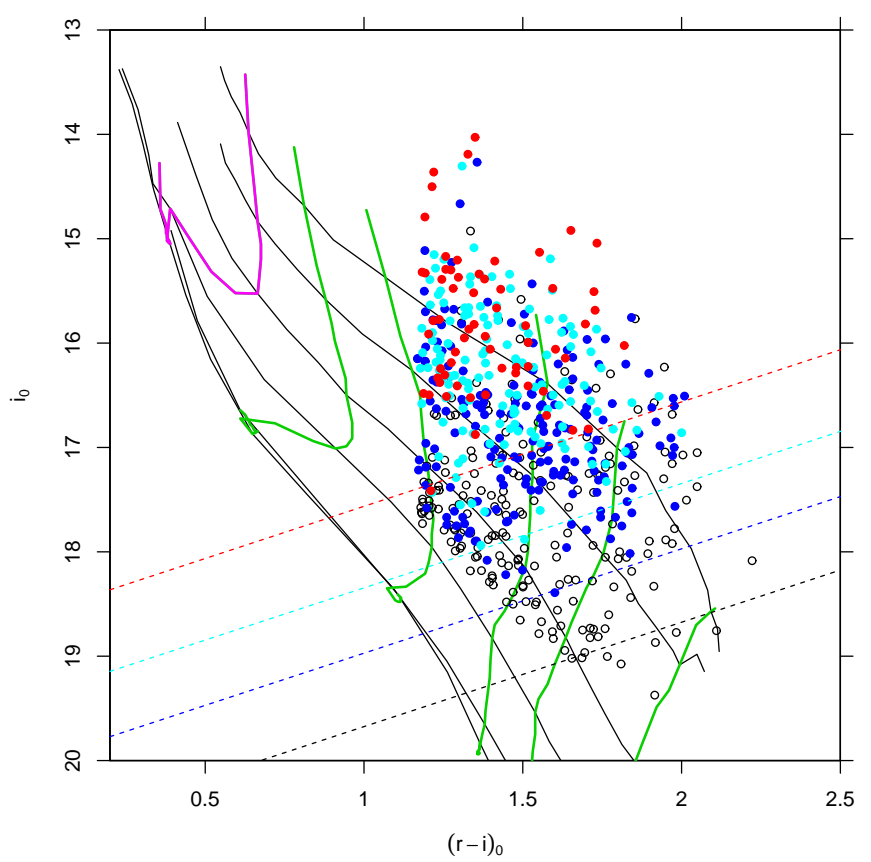

Fig. 14. Dereddened CMD for M stars in final cluster region defined in Fig. 12. Evolutionary tracks and isochrones as in the same figure, but with zero reddening. Colored dots are individually-dereddened $\mathrm{M}$ stars, with color-coded extinction ranges. Black: $0.0<A_{V}<0.8$; blue: $0.8<A_{V}<1.7$; cyan: $1.7<A_{V}<2.5$; red: $2.5<A_{V}<3.5$. Oblique dashed lines indicate completeness limits for the same $A_{V}$ ranges.

proven by the detection of 64 Class 0/I IR sources by Kumar and Anandarao (2010).

We have also studied the spatial distribution of extinction behind the cluster, averaged over the line of sight until large distances. This helped in understanding the environment in which NGC 6530 is found. The average extinction is well approximated by the average $J-H$ color, computed in small spatial bins. The same approach was successfully taken in Paper I, and earlier in Damiani et al. (2016). The map of average $J-H$ is shown in Fig. 15, from which one may infer extinction, being $A_{V} \sim 9.5 \times(J-H-0.5)$. There is a very good agreement between the zones with the largest extinction from this map, and those with lowest source density from the Gaia density map of Fig. 1. The regions close to the Hourglass are confirmed to be among the most extincted sightlines toward NGC 6530 and the Lagoon nebula. Behind the cluster core there is only a moderate amount of extinction, compared to the Hourglass, and also to the outer regions of the nebula, which appear to bifurcate to the north and south of the cluster core (and the Chandra FOVs). The region southeast of core, near the bright rim where also a number of cluster members are found, is also one of large extinction. Otherwise, the eastern part of the cluster (farthest from the Galactic plane) does not seem to be bound by a thick dust wall, but relatively clear, and the overall morphology of dust suggests that the cluster lies in a cavity carved inside a thick dust envelope, which has already broken on the side opposite with respect to the Galactic plane: these were likely the lowest-density parts of the original dust cloud, and the first ones which were dissipated.

Already very close to the cluster core, the line-of-sight extinction drops to low enough values that distant giants begin to be detectable (red dots in the figure). NGC 6530 and the Lagoon nebula are projected onto the Galactic Bulge, being only $\sim 6^{\circ}$ away from the Galactic center. Giants are found in large

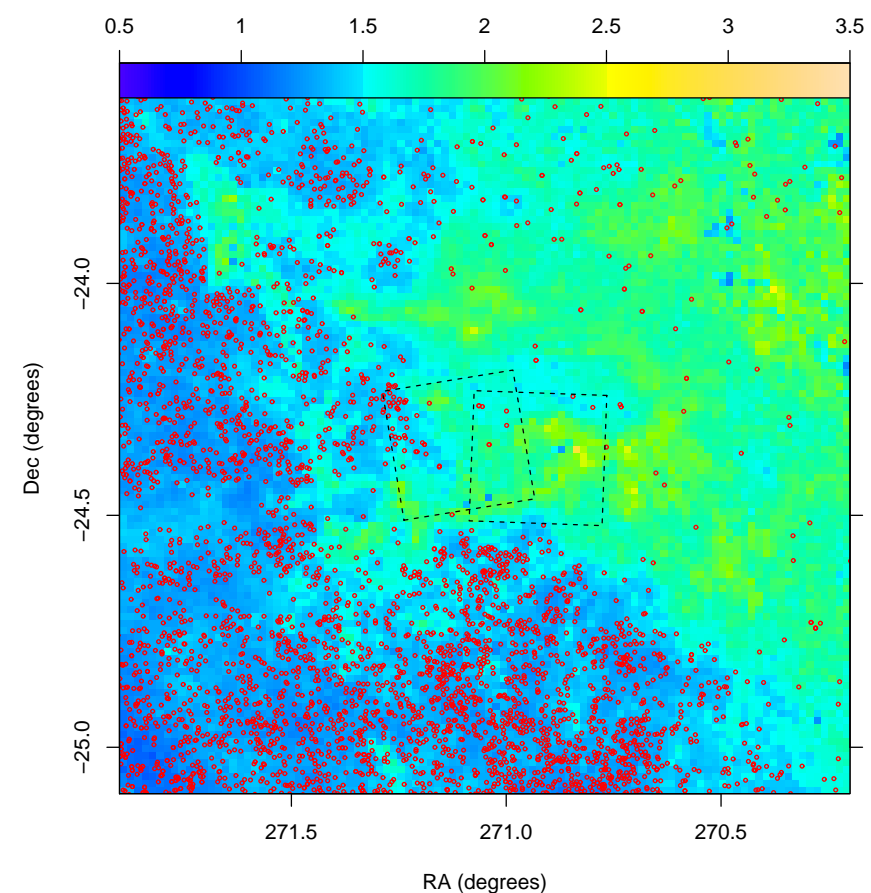

Fig. 15. Map of mean $(J-H)$ color (color scale shown in top axis). Red circles are high-reddening $M$ stars (giants) from Figs. 4-a,b,c. Dashed squares are the Chandra FOVs.

numbers only in the regions with relatively lower densities (i.e., not exceeding 10-15 mag in $V$ ), in agreement with their $A_{V}$ values that can be derived from the diagrams in Figs. 4-a,b,c; their spatial distribution matches the distribution of extinction very well, providing us with a very coherent picture. Especially the southeastern parts of NGC 6530 are surrounded by lower-density sightlines. Therefore, the corresponding obscuring dust is likely a protruding cloud, with very little dense material all around. Lacking pressure balance against the (radiative and mechanical) push from the massive stars in NGC 6530, the southeastern part of the cloud will probably disperse soon after it ceases to form new stars.

\subsection{Ages of members}

After placing $\mathrm{M}$ stars individually on the extinction-corrected CMD, we may determine more accurately their ages by comparison with BHAC isochrones. These age values are only valid for stars at the assumed distance (no selection on parallax is made here). We therefore obtain some indications on the starformation history in the Lagoon nebula from the spatial distributions of M-type members in several age ranges, shown in Fig. 16. Very few, if any, cluster members are found to be older than $4 \mathrm{Myr}$ in panel $\mathrm{d}$. This statement must be accompanied by the caveat that we are only able to find the most massive Mtype stars in this age range, as also predicted by the MDA diagrams of Fig. 3. For younger ages our results are more reliable (panels a-c): star formation appears to have begun in the region common to the two Chandra FOVs, close to the most massive cluster member (the $\mathrm{O} 4 \mathrm{star} 9 \mathrm{Sgr}$ ); then, it both continued in the same place, and propagated to the east; afterwards, star formation near to 9 Sgr stopped, while it became more active to the east. We are unfortunately unable to find $M$ stars in the Hourglass region using the VPHAS + data, although stars embedded in the Hourglass nebula itself are likely to be very young. Stars in the western subgroups appear to have intermediate ages. 

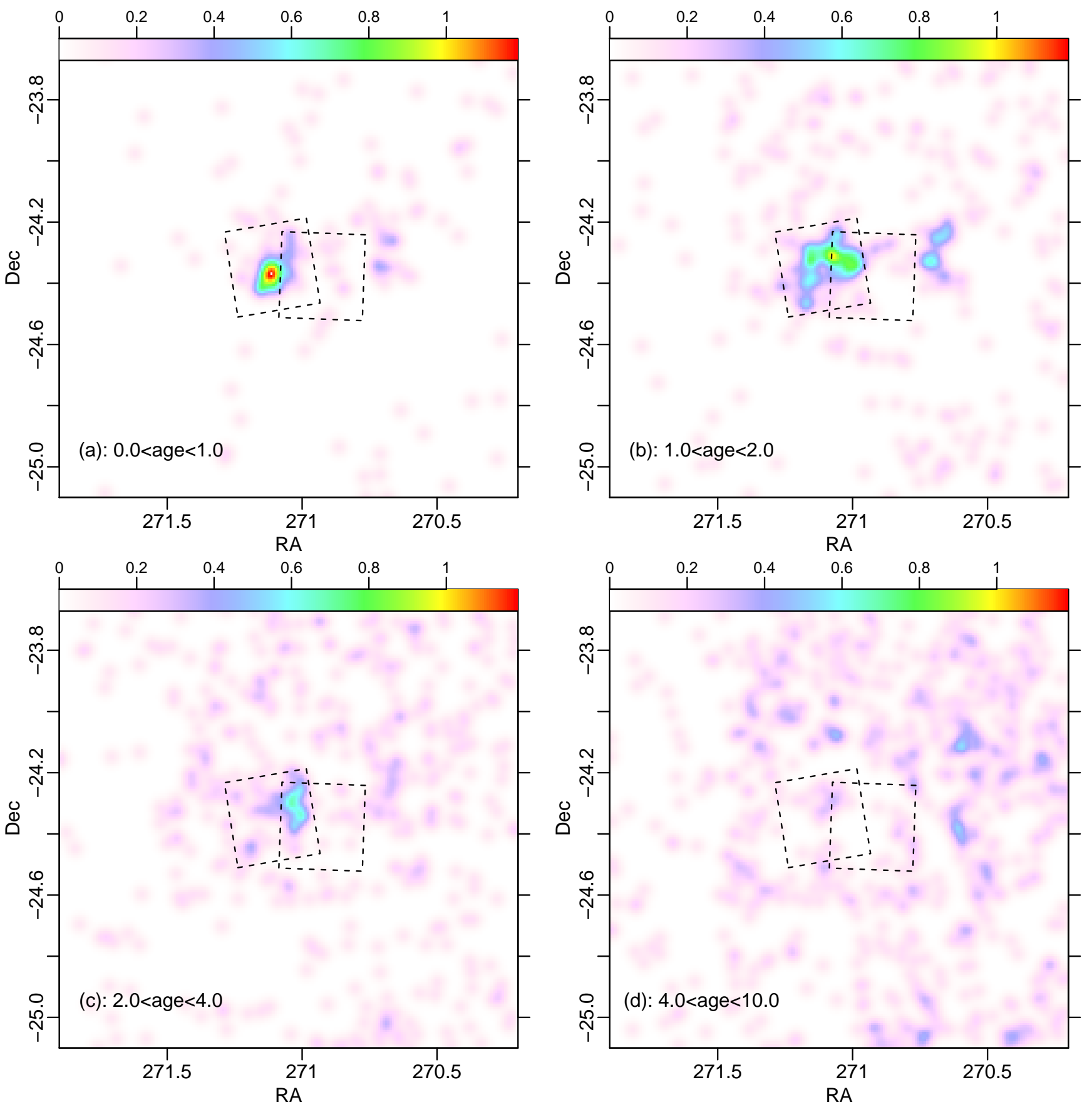

Fig. 16. Spatial distributions of $M$ stars in different age ranges, as indicated (in Myr).

Such a complex pattern of formation events with time and space might justify why different authors have come to different conclusions on the formation history in NGC 6530, on the basis of different studied samples, and therefore under different selection biases (see, e.g., Kalari et al. 2015). Also in the present work, the extinction-related bias as discussed above, as well as the lack of suitable optical photometry near the Hourglass, prevent to obtain a definitive picture of the star-formation history in the Lagoon nebula.

\subsection{Cluster dynamics}

We have presented in Sect. 3.4 the proper-motion distributions of both the cluster and the surrounding field stars. We here consider the average cluster proper motion, in an attempt to estimate the path followed by the cluster in the last few Myrs. The median proper motion of the X-ray detected Gaia members in the cluster region (a highly reliable sample) is $\left(\mu_{\alpha}, \mu_{\delta}\right)=$ $(1.268,-1.996)$ mas $^{-1}{ }^{-1}$. Therefore, the relative motion with respect to the average Galactic-plane population in the same direction is $\left(\Delta \mu_{\alpha}, \Delta \mu_{\delta}\right)=(1.520,-0.201) \mathrm{mas} \mathrm{yr}^{-1}$. This relative motion implies that the cluster has traveled a significant distance in the last few Myrs. Figure 17 shows this path, and demonstrates that NGC 6530 is likely to have crossed the Galactic plane around $4 \mathrm{Myr}$ ago. It is intriguing that this is approximately the age of the oldest members whose age we can measure. A very plausible scenario is therefore that the encounter between the NGC6530 parent molecular cloud, 
coming from above the plane, and the Galactic plane itself, did generate a rapid and strong compression of the cloud, leading to rapid star formation while the cloud continued its travel toward negative Galactic latitudes. Collisions between molecular clouds and the Galactic disk are modeled by Tenorio-Tagle et al. (1987) and Comerón \& Torra (1992), and are suggested to lead to triggered star formation by (for example) Comerón (2001).

For the same member sample, the observed ranges of $\mu_{\alpha}$ and $\mu_{\delta}\left(2.5 \mathrm{mas} \mathrm{yr}^{-1}\right.$ for both) are about ten times larger than the median $\mu_{\alpha}, \mu_{\delta}$ errors. This opens up the possibility that the internal cluster dynamics might be resolved with these data. We have therefore computed the spatial distributions of $\mu_{\alpha}$ for Gaia

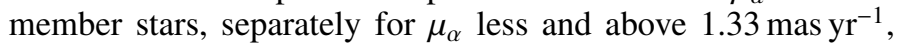
and show their (normalized) difference in Fig. 18a. Here yellow indicates a relative preponderance of stars moving toward right (lower RA) with respect to the mean cluster motion, while blue indicates stars in relative motion toward left. The same was

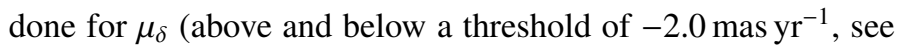
Fig. 18b). Also in this latter case, yellow indicates stars in relative motion toward negative Dec, and blue the opposite. We observe that there is near symmetry of these relative motions with respect to the cluster core (the center of the left-hand Chandra FOV), and the sense of motion indicates expansion away from this center, along both RA and Dec. Near the cluster periphery different motion patterns are instead found, both close to the Hourglass and in the western group, which both move toward east. It should be remarked however, that such motions are averages with non-negligible internal spread (due to both true random motion and measurement errors), as seen from the individual distributions used to produce the difference images in Fig. 18; these are also shown in the same figure by red and blue contours, which largely overlap. Similar results on expansion in NGC 6530 have recently been obtained by Kuhn et al. (2019) and Wright et al. (2018).

\subsection{Member statistics and contamination}

Results from the previous section have shown that the different member selection methods used are mutually complementary, each one being most effective on some parts of the large parameter space (range of masses, ages, extinction values, importance of disk- and accretion-related phenomena) occupied by NGC 6530 members. In this section we examine this more quantitatively, and try to estimate the level of contamination which affects subsamples found by any particular method.

Figure 19 is a Venn diagram showing the intersection between the four selection criteria employed. For simplicity, we have put in a single sample (CTTS) all stars selected by either $\mathrm{H} \alpha$ emission, NIR- or UV-excesses. All samples are spatially limited to the final cluster region. The M-star and CTTS samples include the photometric threshold of Sect. 3.5. The ordinary Venn diagram applied to four samples with such a complex pattern of overlaps does not permit to show graphically all pairwise intersections: this is the case of the diagonally opposite samples (e.g., X-ray and CTTS samples), whose exclusive intersection (i.e., with no stars in common to the Gaia and M-star samples) cannot be represented graphically. Therefore, we have "folded" around its corners the diagram to show such intersections, so that, for example, the lower-left dotted circle duplicates the CTTS sample in the upper-right solid circle, and its exclusive intersection with the X-ray sample (39 objects) is made visualizable. The same holds of course for all four samples. By symmetry, the X-ray-CTTS intersection is duplicated in the upper-right corner, and the number of objects shown there refer to exactly

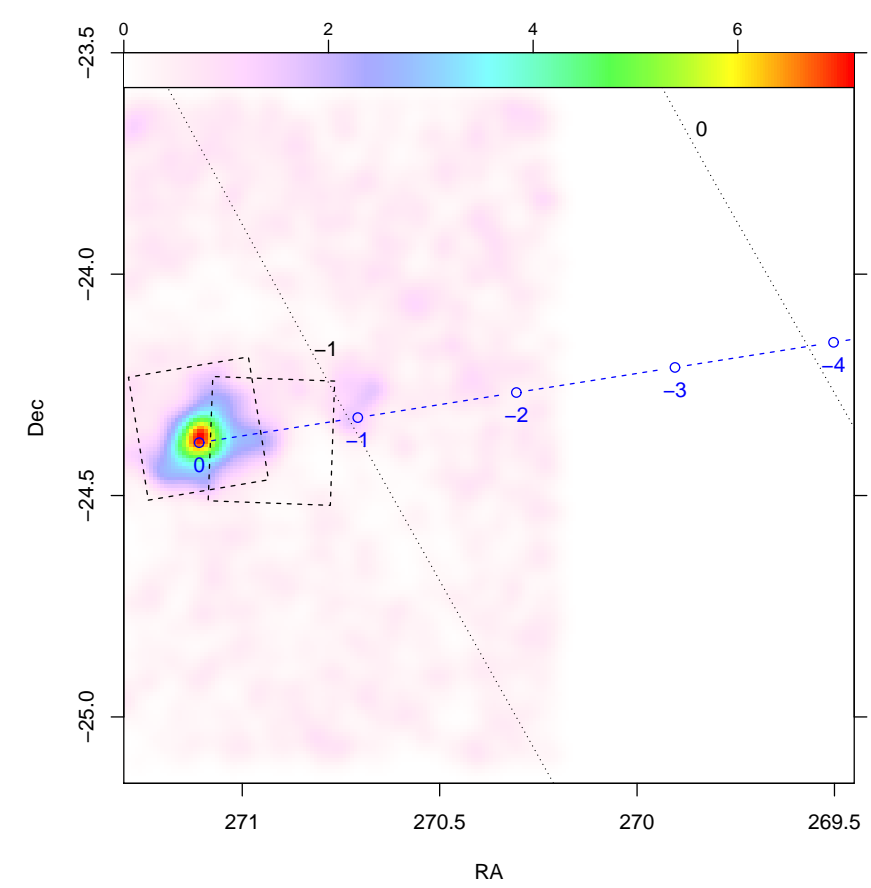

Fig. 17. Smoothed spatial distribution of Gaia members, plus backprojected mean cluster position, from about $4 \mathrm{Myr}$ ago to now (blue dot-dashed line). Blue labels indicate time in Myr. Dotted black lines indicate constant Galactic latitude $b$, as labeled.

the same objects in the opposite corner: when computing a grand total (3675 stars), those objects must only be counted once. They are indicated twice in the figure, however, to make the sum of numbers inside each circle equal to the total number in the respective sample (indicated just outside each circle). The complete photometry of the 3675 candidate members is reported in Table 1, together with their classification as optical/NIR excess stars, M stars, X-ray sources and Gaia members.

Stars fulfilling more membership criteria are betterconfidence members. However, the diagram shows that the number of stars fulfilling all criteria is very small (three objects), so being extremely conservative in the selection would give meaningless results. Also very small subsamples fulfill three criteria. We therefore consider all stars with at least two criteria as reliable members individually (711 stars). The largest overlap between two subsamples is that between Gaia and X-rays, with $292(251+16+22+3)$ common objects. Then, M-stars and $\mathrm{X}$-rays have $148(109+20+16+3)$ stars in common, M-stars and CTTS have $126(96+20+7+3)$ stars in common, CTTS and Gaia have $61(22+7+3+29)$ stars in common, Gaia and M-stars have $66(40+16+3+7)$ stars in common, and X-rays and CTTS have $84(39+20+3+22)$ stars in common. These relatively small numbers confirm the complementarity between selection criteria.

Statistically, also stars with a single membership criterion (i.e., $839 \mathrm{M}$ stars, 337 CTTS stars, 1296 X-ray sources, and 571 Gaia members, see Fig. 19) are members in a given percentage, as was qualitatively shown in Figs. 11-c,d. The number of non-member contaminants may be estimated by applying the same selection to the reference region surrounding the final cluster region, and scaling for the respective sky areas. The estimated contaminants are thus 525, 89, and 333 for the M-star, CTTS, and Gaia samples, respectively $(62.6 \%, 26.4 \%$, and $58.3 \%$ of the total single-criteria samples, respectively). Therefore, the estimated net number of members selected exclusively by one 

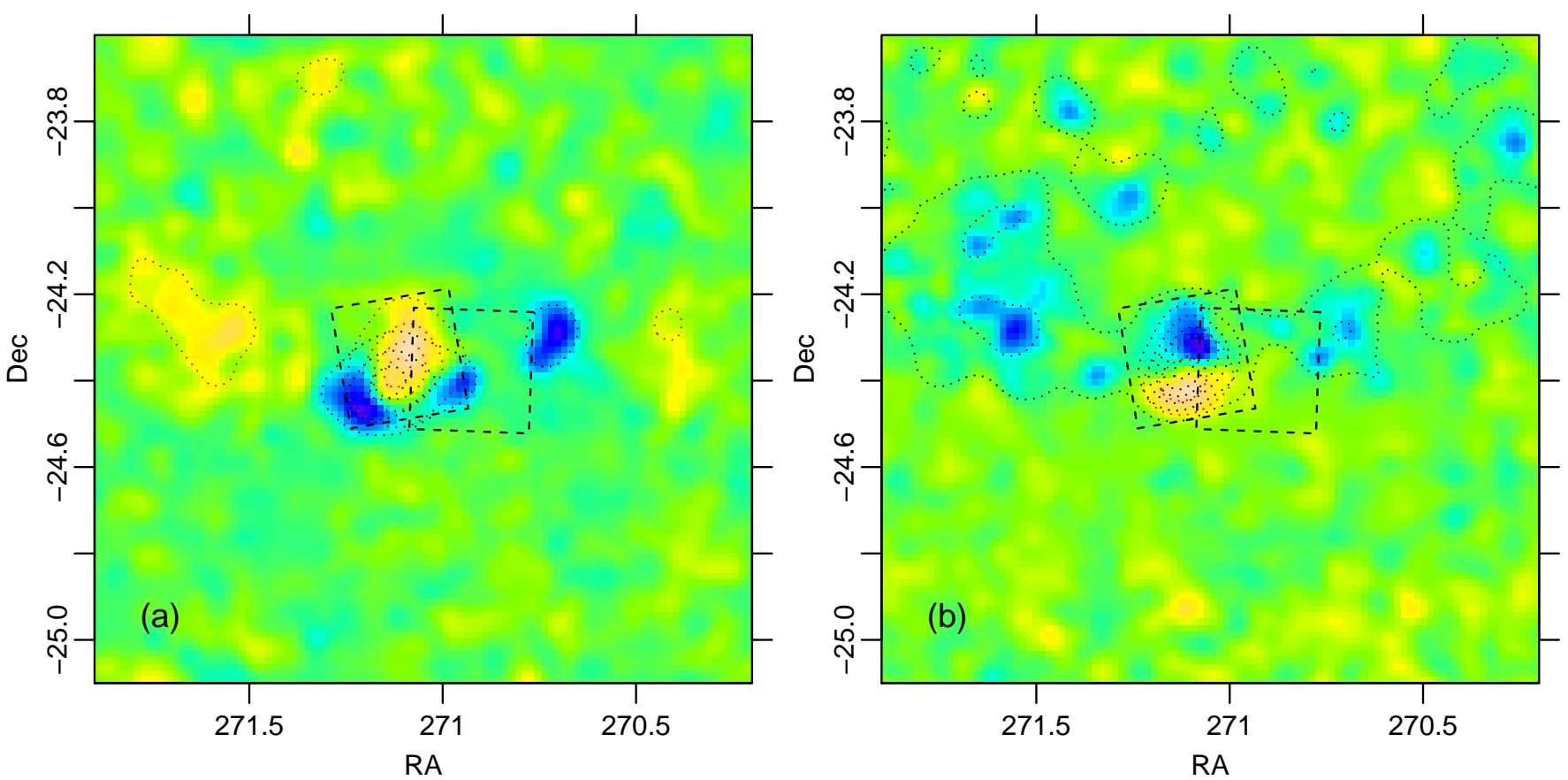

Fig. 18. Panel a: difference between spatial distributions of Gaia members (according to both proper motion and parallax) with $\mu_{\alpha}$ less and above

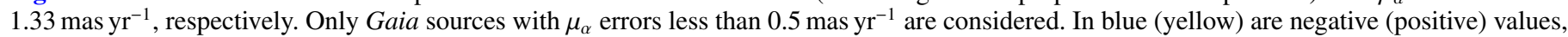
with green indicating zero difference. Dashed squares are the Chandra FOVs. The red and black dotted contours describe the original distributions in the two $\mu_{\alpha}$ ranges, whose difference is shown by the map. Panel $b$ : same as panel a, but showing the difference between distributions of stars

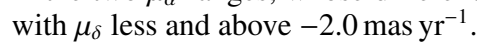

Table 1. Optical/NIR photometry for all candidate members of NGC 6530.

\begin{tabular}{|c|c|c|c|c|c|c|c|c|c|c|c|c|c|c|c|c|c|}
\hline $\begin{array}{c}\text { Seq } \\
\text { no. }\end{array}$ & $\begin{array}{c}\text { RA } \\
(\mathrm{J} 2000)\end{array}$ & $\begin{array}{c}\text { Dec } \\
(\mathrm{J} 2000)\end{array}$ & VPHAS Id & $i$ & $r-i$ & $r-H \alpha$ & $g-r$ & $u-g$ & $J$ & $J-H$ & $H-K$ & $\begin{array}{l}\text { IR } \\
\text { exc. }\end{array}$ & $\begin{array}{l}\mathrm{H} \alpha \\
\text { exc. }\end{array}$ & $\begin{array}{l}\text { UV } \\
\text { exc. }\end{array}$ & $\begin{array}{c}\mathrm{M} \\
\text { star }\end{array}$ & $\begin{array}{c}\text { X-ray } \\
\text { det. }\end{array}$ & Gaia \\
\hline 1 & 270.75503 & -24.47542 & $\mathrm{~J} 180301.2-242831.4$ & 20.95 & 1.80 & & & & 17.14 & 0.75 & 0.27 & & & & $\mathrm{Y}$ & & \\
\hline 2 & 270.77272 & -24.45467 & $\mathrm{~J} 180305.5-242716.8$ & 20.45 & 1.73 & & & & 16.78 & 0.91 & 0.35 & & & & $\mathrm{Y}$ & & \\
\hline 3 & 270.86873 & -24.45169 & $\mathrm{~J} 180328.5-242706.1$ & & & & & & & & & & & & & & $\mathrm{Y}$ \\
\hline 4 & 270.82115 & -24.49788 & $\mathrm{~J} 180317.1-242952.3$ & & & & & & & & & & & & & & $\mathrm{Y}$ \\
\hline 5 & 270.85809 & -24.46839 & $\mathrm{~J} 180325.9-242806.2$ & & & & & 0.94 & & & & & & & & $\mathrm{Y}$ & \\
\hline 6 & 270.82926 & -24.46857 & $\mathrm{~J} 180319.0-242806.8$ & & & & & & & & & & & & & $\mathrm{Y}$ & \\
\hline 7 & 270.76139 & -24.52889 & $\mathrm{~J} 180302.7-243143.9$ & 20.11 & 1.48 & & & & 14.52 & 1.75 & 0.81 & $\mathrm{Y}$ & & & & & \\
\hline 8 & 270.82775 & -24.42138 & $\mathrm{~J} 180318.7-242516.9$ & 20.77 & & & & & 17.32 & 0.80 & 0.22 & & & & & $\mathrm{Y}$ & \\
\hline 9 & 270.81253 & -24.47325 & $\mathrm{~J} 180315.0-242823.6$ & 19.61 & 1.66 & & 1.65 & & & & & & & & $\mathrm{Y}$ & & \\
\hline 10 & 270.73951 & -24.54836 & $\mathrm{~J} 180257.5-243254.0$ & 19.85 & 1.49 & 0.67 & 1.83 & & & & & & & & $\mathrm{Y}$ & & \\
\hline 11 & 270.76578 & -24.51855 & $\mathrm{~J} 180303.8-243106.7$ & 18.25 & 1.02 & 0.59 & 1.81 & & & & & & & & & & $\mathrm{Y}$ \\
\hline 12 & 270.72789 & -24.50358 & $\mathrm{~J} 180254.7-243012.8$ & 18.10 & & & 1.66 & & & & & & & & & & $\mathrm{Y}$ \\
\hline 13 & 270.77432 & -24.52539 & $\mathrm{~J} 180305.8-243131.3$ & 20.81 & 1.73 & & & & 17.43 & 0.72 & 0.17 & & & & $\mathrm{Y}$ & & \\
\hline 14 & 270.77804 & -24.49452 & $\mathrm{~J} 180306.7-242940.2$ & 18.39 & & 0.51 & 2.22 & & 14.67 & 0.82 & 0.33 & & & & & & $\mathrm{Y}$ \\
\hline 15 & 270.81611 & -24.50425 & $\mathrm{~J} 180315.9-243015.2$ & 16.06 & & & 1.49 & 1.39 & & & & & & & & & $\mathrm{Y}$ \\
\hline 16 & 270.76885 & -24.41684 & $\mathrm{~J} 180304.5-242500.6$ & 20.20 & 1.68 & & & & 16.51 & 0.96 & 0.36 & & & & $\mathrm{Y}$ & & \\
\hline 17 & 270.84740 & -24.48966 & $\mathrm{~J} 180323.4-242922.7$ & & & & & & 14.48 & 0.98 & 0.43 & & & & & & $\mathrm{Y}$ \\
\hline 18 & 270.58659 & -24.52602 & $\mathrm{~J} 180220.8-243133.7$ & 20.27 & 1.87 & 0.94 & & & 16.27 & 0.90 & 0.39 & & & & $\mathrm{Y}$ & & \\
\hline 19 & 270.83647 & -24.44672 & $\mathrm{~J} 180320.8-242648.2$ & 20.49 & 1.94 & & & & 15.07 & 1.36 & 0.56 & & & & & $\mathrm{Y}$ & \\
\hline 20 & 270.77585 & -24.48878 & $\mathrm{~J} 180306.2-242919.5$ & & & & & & 13.82 & 0.70 & 0.31 & & & & & & $\mathrm{Y}$ \\
\hline
\end{tabular}

Notes. Full table available at the CDS.

criterion are 314, 248, and 238 for the M-star, CTTS, and Gaia criteria, respectively. Unfortunately this cannot be done for the $\mathrm{X}$-ray sample, there being no Chandra data for the reference region, but in similar X-ray pointings the number of field X-ray sources is of order of $\sim 100$. These unrelated sources are however partly absorbed by those with no optical-NIR counterpart, which we excluded from our analysis (see Sect. 2.3), so that it is probably unnecessary to subtract them here from the X-ray member count. The total net number of NGC 6530 members estimated with all our methods becomes therefore of 2728 stars, down to $0.2-0.4 M_{\odot}$, the exact limit being dependent on individual stellar ages and extinction. If we add the $671 \mathrm{X}$-ray detections without optical counterpart, strongly clustered inside the Lagoon nebula, we obtain a total population of 3399 stars.

A CMD of all final members is shown in Fig. 20. The same qualitative considerations apply as for the CMD of 


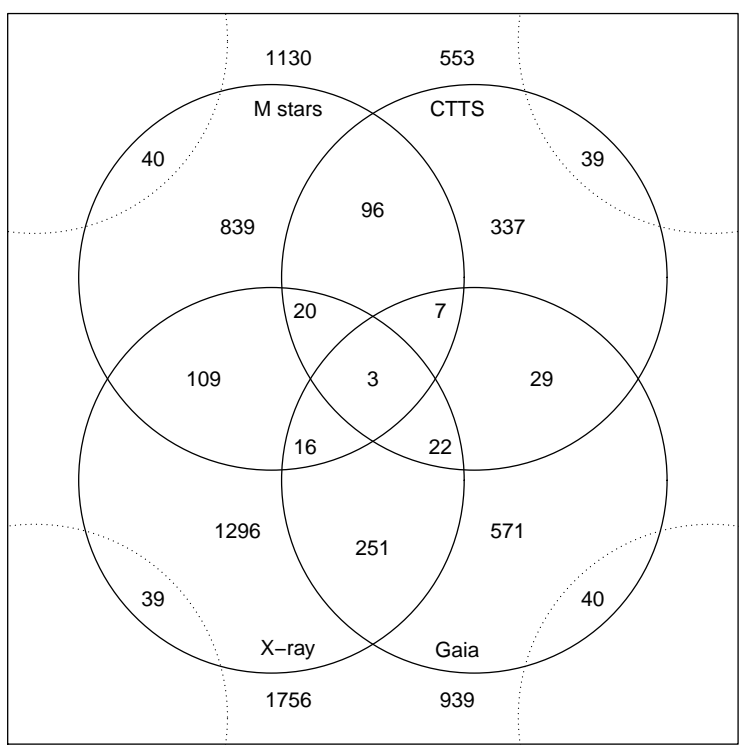

Fig. 19. Venn diagram showing the intersections among the different member subsamples. The total number of candidate members for each subsample is shown outside the corresponding circle, near its label. The diagram wraps around its corners, in order to show the exclusive intersections between X-ray and CTTS samples, and between Gaia and Mstar samples.

Gaia-detected members of Fig. 8 (Sect. 3.5). The M-star and CTTS samples are most contaminated by field stars near the lower envelope of datapoints (between the 10 and $30 \mathrm{Myr}$ isochrones). This is shown both by comparison with the CMD of the reference field (not shown), and by the lack of clustering of stars at the oldest apparent ages (Fig. 16). These stars are kept here for completeness, but their individual membership needs a more careful assessment. It is noteworthy that, after considering all corrections for contamination, the net percentages of CTTSs among all members ((553-89)/2728, or 17\%) or that among M stars (126/(1130-525), or 21\%) are low compared to analogous percentages for other $1-2 \mathrm{Myr}$ clusters $(60-80 \%$ according to Haisch et al. 2001). This might be related to disk photoevaporation caused by the winds and radiation from massive stars, as suggested by Guarcello et al. (2007) for the young cluster NGC 6611.

\section{Conclusions}

By combining various techniques for member selection, complementary to each other, we have assembled a list of 3675 candidate NGC 6530 members down to approximately $0.3 M_{\odot}$. Of them, only 711 fulfill more than one membership criterion, but we estimated that the total net number of members in our list is slightly over 2700 . Among the single-criterion subsamples, the X-ray sample was estimated to be the least contaminated by field stars, while contamination ranges from $26 \%$ to $62 \%$ for the M-star, CTTS and Gaia selected subsamples. Nevertheless, the statistical properties of the NGC 6530 population are very well defined, since even though the population in our different subsamples do not coincide star-by-star, they often exhibit similar general properties, which demonstrate the robustness of the results obtained. Results from our newly-developed method for selecting M-type cluster members are validated by the new Gaia DR2 data, which in turn agree very well with the X-ray selected members.

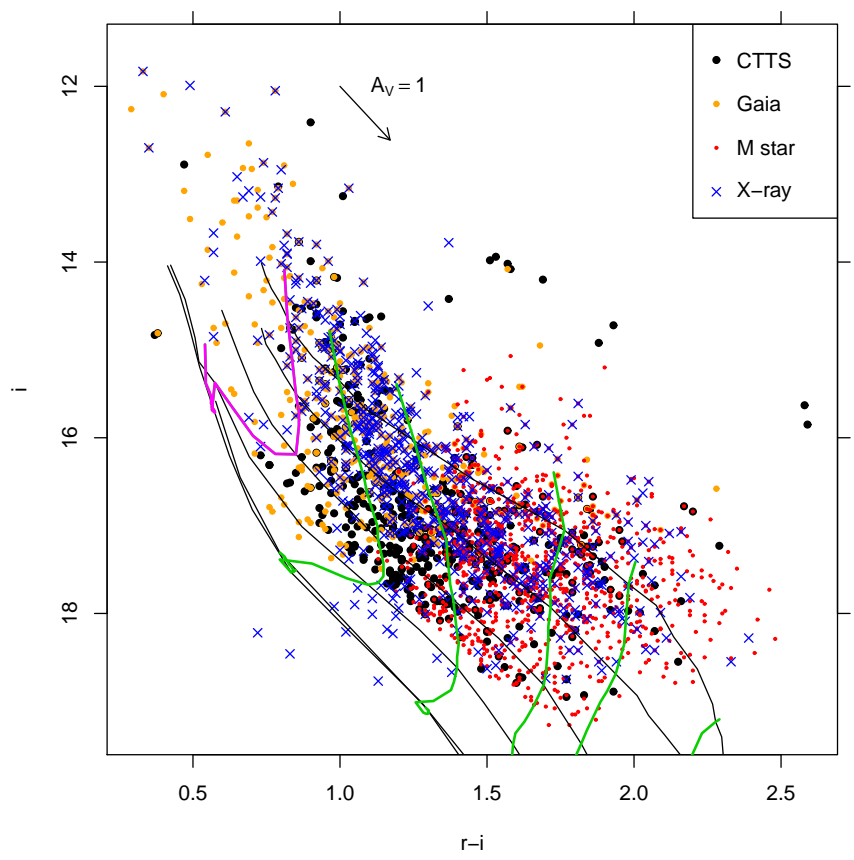

Fig. 20. CMD of final candidate members. Track and isochrones (ages of $1,3,10,30,100 \mathrm{Myr}$ and $10 \mathrm{Gyr}$ ) as in Fig. 8 .

The Gaia distance to NGC 6530 was found to be $1325 \mathrm{pc}$, which considering the systematic error is compatible with our previous determination $(1250 \mathrm{pc})$. The NGC 6530 morphology was confirmed to be complex, as from previous studies, with a cluster core that contains the bulk of members, and secondary concentrations of members in known regions (the Hourglass nebula, the M8-East region) and anonymous, small groups (two groups in the N-W part, close to the stars 7 Sgr and HD 164536). There is no diffuse population of members all around the cluster.

Thanks to our new method for selecting M-type members, whose extinction can be individually determined, we found that member stars lie behind a variable amount of obscuration, with a defined spatial pattern. The literature value of extinction $\left(A_{V}=\right.$ 1.08, Sung et al. 2000) corresponds to the lower bound to the $A_{V}$ range found here. The maximum extinction is not well defined because of our limiting magnitude, but may occasionally be very large (as in the case of the well-known embedded protostar M8-East IR).

Isochronal ages also showed a complex spatial pattern, with a general trends of age decreasing from center toward (skyprojected) periphery. There are young (and extincted) stars even close to the (otherwise older and little obscured) cluster core, suggesting that star formation has also proceeded along the line of sight, toward deeper layers inside the molecular cloud. The western regions are apparently not involved in this sequence, and have an independent star-formation history from the rest of NGC 6530. There is no significant population (if at all) of cluster stars older than $5 \mathrm{Myr}$.

The Gaia dynamical data showed that the NGC 6530 cluster and its parental cloud crossed the Galactic plane about $4 \mathrm{Myr}$ ago. Therefore, that event might have been the main trigger of all star-formation phenomena thereafter. The NGC 6530 parent molecular cloud (whose illuminated part is the Lagoon nebula) is now traveling toward regions of lower ambient density, and we found evidence that its border at the lowest (negative) latitudes $b$ is being disrupted, probably by the winds and radiation from the massive OB stars in the cluster. 
The precision of Gaia proper-motion data also enabled us to partially resolve the internal cluster dynamics, and provided indications that the bulk of the cluster is expanding away from its center. It is expected that Gaia data from later data releases will permit even more detailed studies of the NGC 6530 internal dynamics. Also, deeper photometric surveys (e.g., from LSST) will help to discover the entire NGC 6530 population down to the substellar limit.

Acknowledgements. We wish to thank an anonymous referee for his/her helpful suggestions. Based on data products from observations made with ESO Telescopes at the La Silla Paranal Observatory under program ID 177.D3023, as part of the VST Photometric $\mathrm{H} \alpha$ Survey of the Southern Galactic plane and Bulge (VPHAS+, www.vphas.eu). This work has made use of data from the European Space Agency (ESA) mission Gaia (https://www cosmos.esa.int/gaia), processed by the Gaia Data Processing and Analysis Consortium (DPAC, https://www. cosmos.esa.int/web/gaia/dpac/ consortium). Funding for the DPAC has been provided by national institutions, in particular the institutions participating in the Gaia Multilateral Agreement. Also based on data products from VVV Survey observations made with the VISTA telescope at the ESO Paranal Observatory under program ID 179.B2002. The scientific results reported in this article are also based on observations made by the Chandra X-ray Observatory. This publication makes use of data products from the Two Micron All Sky Survey, which is a joint project of the University of Massachusetts and the Infrared Processing and Analysis Center/California Institute of Technology, funded by the National Aeronautics and Space Administration and the National Science Foundation. This research makes use of the SIMBAD database and the Vizier catalog service, operated at CDS, Strasbourg, France. We also make heavy use of R: A language and environment for statistical computing. R Foundation for Statistical Computing, Vienna Austria. (http://www.R-project.org/).

\section{References}

Bailer-Jones, C. A. L., Rybizki, J., Fouesneau, M., Mantelet, G., \& Andrae, R. 2018, AJ, 156, 58

Baraffe, I., Homeier, D., Allard, F., \& Chabrier, G. 2015, A\&A, 577, A42

Chambers, K. C., Magnier, E. A., Metcalfe, N., et al. 2016, ArXiv e-prints [arXiv:1612.05560]

Comerón, F. 2001, From Darkness to Light: Origin and Evolution of Young Stellar Clusters, 243, 807
Comerón, F., \& Torra, J. 1992, A\&A, 261, 94

Damiani, F. 2018, A\&A, 615, A148

Damiani, F., Flaccomio, E., Micela, G., et al. 2004, ApJ, 608, 781

Damiani, F., Prisinzano, L., Micela, G., \& Sciortino, S. 2006a, A\&A, 459, 477

Damiani, F., Micela, G., Sciortino, S., et al. 2006b, A\&A, 460, 133

Damiani, F., Micela, G., \& Sciortino, S. 2016, A\&A, 596, A82

Damiani, F., Bonito, R., Prisinzano, L., et al. 2017a, A\&A, 604, A135

Damiani, F., Pillitteri, I., \& Prisinzano, L. 2017b, A\&A, 602, A115

Drew, J. E., Gonzalez-Solares, E., Greimel, R., et al. 2014, MNRAS, 440, 2036

Feigelson, E. D., \& Montmerle, T. 1999, ARA\&A, 37, 363

Feigelson, E. D., Townsley, L. K., Broos, P. S., et al. 2013, ApJS, 209, 26

Gaia Collaboration (Prusti, T., et al.) 2016, A\&A, 595, A1

Gaia Collaboration (Brown, A. G. A., et al.) 2018, A\&A, 616, A1

Guarcello, M. G., Prisinzano, L., Micela, G., et al. 2007, A\&A, 462, 245

Haisch, Jr., K. E., Lada, E. A., \& Lada, C. J. 2001, ApJ, 553, L153

Lawrence, A., Warren, S. J., Almaini, O., et al. 2007, MNRAS, 379, 1599

Kalari, V. M., Vink, J. S., Drew, J. E., et al. 2015, MNRAS, 453, 1026

King, R. R., Naylor, T., Broos, P. S., Getman, K. V., \& Feigelson, E. D. 2013 ApJ, 209, 28

Kuhn, M. A., Getman, K. V., Broos, P. S., Townsley, L. K., \& Feigelson, E. D. 2013, ApJS, 209, 27

Kuhn, M. A., Feigelson, E. D., Getman, K. V., et al. 2014, ApJ, 787, 107

Kuhn, M. A., Getman, K. V., \& Feigelson, E. D. 2015, ApJ, 802, 60

Kuhn, M. A., Hillenbrand, L. A., Sills, A., Feigelson, E. D., \& Getman, K. V. 2019, ApJ, 870, 32

Kumar, D. L., \& Anandarao, B. G. 2010, MNRAS, 407, 1170

Luri, X., Brown, A. G. A., Sarro, L. M., et al. 2018, A\&A, 619, A9

Minniti, D., Clariá, J. J., Saito, R. K., et al. 2011, Boletín de la Asociación Argentina de Astronomía La Plata Argentina, 54, 265

Prisinzano, L., Damiani, F., Micela, G., \& Sciortino, S. 2005, A\&A, 430, 941

Prisinzano, L., Damiani, F., Micela, G., \& Pillitteri, I. 2007, A\&A, 462, 123

Prisinzano, L., Micela, G., Sciortino, S., Affer, L., \& Damiani, F. 2012, A\&A, 546, A9

Prisinzano, L., Damiani, F., Guarcello, M. G., et al. 2018, A\&A, 617, A63

Siess, L., Dufour, E., \& Forestini, M. 2000, A\&A, 358, 593

Sung, H., Chun, M.-Y., \& Bessell, M. S. 2000, AJ, 120, 333

Tenorio-Tagle, G., Franco, J., Bodenheimer, P., \& Rozyczka, M. 1987, A\&A, 179,219

Tothill, N. F. H., Gagné, M., Stecklum, B., \& Kenworthy, M. A. 2008, Handbook of Star Forming Regions, Volume II, 5, 533

van den Ancker, M. E., The, P. S., Feinstein, A., et al. 1997, A\&AS, 123, 63

Venuti, L., Damiani, F., \& Prisinzano, L. 2019, A\&A, 621, A14

Wright, E. L., Lada, C. J., Fazio, G. G., Low, F. J., \& Kleinmann, D. E. 1977, AJ, 82, 132

Wright, N. J., Jeffries, R. D., Jackson, R. J., et al. 2018, MNRAS, submitted 


\section{Appendix A: VVV-UKIDSS calibration}

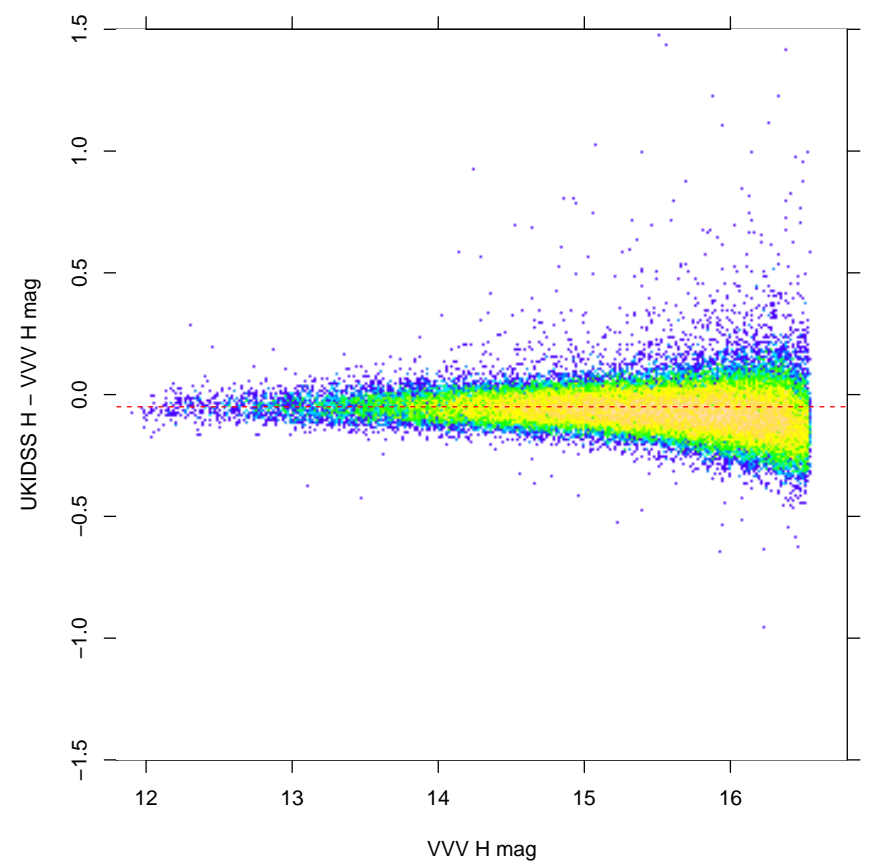

Fig. A.1. Difference in $H$ magnitudes between King-UKIDSS and VVV catalogs, vs. VVV $H$ magnitude. The red dashed line indicate the average difference of $-0.05 \mathrm{mag}$.

Here we examine the photometric differences between the KingUKIDSS and VVV magnitudes in the $H$ and $K$ bands. Only sources with magnitude errors less than $0.1 \mathrm{mag}$ were used in each of the plots. Fig. A.1 presents the comparison for the $H$ band and Fig. A.2 for the $K$ band. The red dashed lines indicate the average difference. No color dependence was found.

\section{Appendix B: Reddening law}

Here we provide some details on the reddening law used in this work, which was as far as possible derived from the

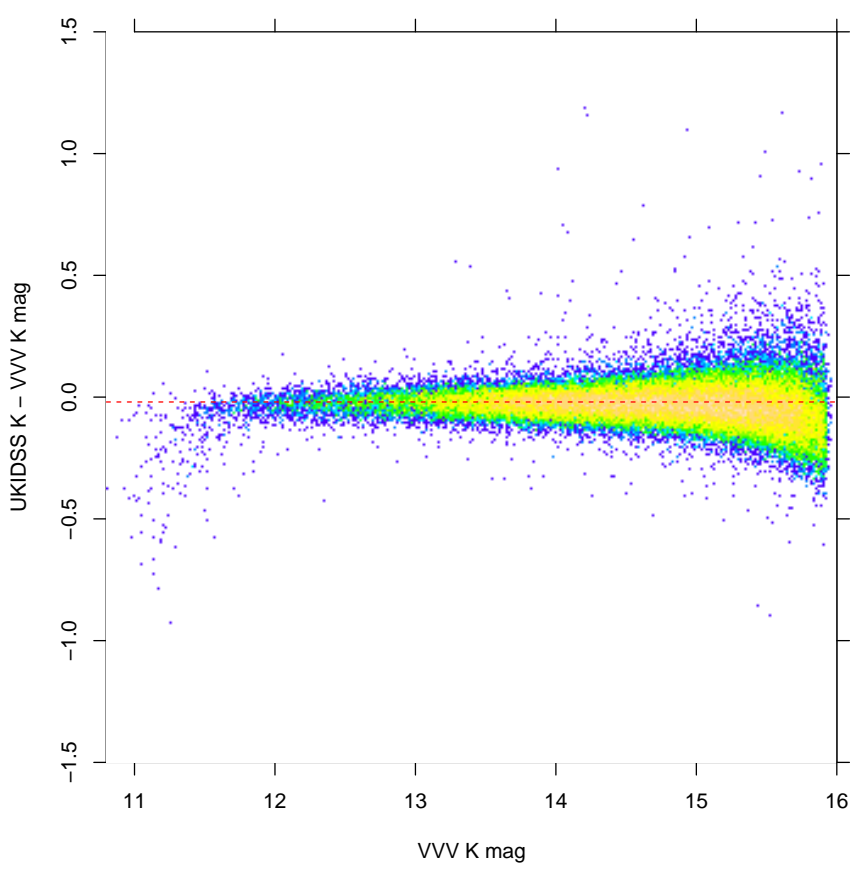

Fig. A.2. Difference in $K$ magnitudes between King-UKIDSS and VVV catalogs, vs. VVV $K$ magnitude. The red dashed line indicate the average difference of $-0.02 \mathrm{mag}$.

photometric data studied here. The bulk of datapoints in panels a,b,c,e of Fig. 4, where the effect of reddening is dominant, define clearly the color-excess ratios $E(r-i) / E(g-r)=0.48$, $E(r-i) / E(i-J)=0.53, E(r-i) / E(i-H)=0.4$, and $E(r-i) / E(H-K)=3.1$, respectively, in the direction of the Lagoon nebula. Moreover, the $(J-H, H-K)$ diagram (not shown) indicates $E(J-H) / E(H-K)=1.9$. These relations are sufficient for the color-based selections made in Sect. 3, but not for estimates of optical extinction or stellar masses from isochrones. Therefore, we need two more relations in order to obtain a complete reddening law and extinction estimates. These were chosen to be $A_{J} / A_{V}=0.282$ and $A_{K} / A_{V}=0.175$ from Rieke and Lebofsky (1985), assuming that any peculiar reddening law has negligible effect in the $J$ and $H$ bands. 